\title{
Nitrate Assimilation in Fusarium fujikuroi Is Controlled by Multiple Levels of Regulation
}

\author{
Andreas Pfannmüller, Jana M. Boysen and Bettina Tudzynski * \\ Molecular Biology and Biotechnology of Fungi, Department of Biology, Institute of Biology and Biotechnology of Plants, \\ University of Münster, Münster, Germany
}

Secondary metabolite production of the phytopathogenic ascomycete fungus Fusarium fujikuroi is greatly influenced by the availability of nitrogen. While favored nitrogen sources such as glutamine and ammonium are used preferentially, the uptake and utilization of nitrate is subject to a regulatory mechanism called nitrogen metabolite repression (NMR). In Aspergillus nidulans, the transcriptional control of the nitrate assimilatory system is carried out by the synergistic action of the nitrate-specific transcription factor NirA and the major nitrogen-responsive regulator AreA. In this study, we identified the main components of the nitrate assimilation system in F. fujikuroi and studied the role of each of them regarding the regulation of the remaining components. We analyzed

\section{OPEN ACCESS}

Edited by:

Sven Krappmann,

University of Erlangen-Nuremberg,

Germany

Reviewed by:

Ozgur Bayram,

Maynooth University, Ireland

Roberto Silva,

University of São Paulo, Brazil

${ }^{*}$ Correspondence:

Bettina Tudzynsk

tudzynski@uni-muenster.de

Specialty section:

This article was submitted to Fungi and Their Interactions,

a section of the journal

Frontiers in Microbiology

Received: 20 December 2016 Accepted: 23 February 2017 Published: 14 March 2017

Citation:

Pfannmüller $A$, Boysen JM and Tudzynski B (2017) Nitrate Assimilation in Fusarium fujikuroi Is

Controlled by Multiple Levels of Regulation. Front. Microbiol. 8:381. doi: 10.3389/fmicb.2017.00381 mutants with deletions of the nitrate-specific activator NirA, the nitrate reductase (NR), the nitrite reductase $(\mathrm{NiR})$ and the nitrate transporter NrtA. We show that NirA controls the transcription of the nitrate assimilatory genes NIAD, NIIA, and NRTA in the presence of nitrate, and that the global nitrogen regulator AreA is obligatory for expression of most, but not all NirA target genes (NIAD). By transforming a NirA-GFP fusion construct into the $\triangle N I A D, \triangle N R T A$, and $\triangle A R E A$ mutant backgrounds we revealed that NirA was dispersed in the cytosol when grown in the presence of glutamine, but rapidly sorted to the nucleus when nitrate was added. Interestingly, the rapid and nitrate-induced nuclear translocation of NirA was observed also in the $\triangle A R E A$ and $\triangle N R T A$ mutants, but not in $\triangle N I A D$, suggesting that the fungus is able to directly sense nitrate in an AreA- and NrtA-independent, but NR-dependent manner.

Keywords: Fusarium, nitrate reductase, NirA, AreA, nitrate sensing, nitrogen metabolite repression, gene regulation

\section{INTRODUCTION}

The phytopathogenic ascomycete fungus Fusarium fujikuroi produces a broad spectrum of interesting secondary metabolites (SM) including the phytohormones gibberellins (GA) and various pigments and mycotoxins. Biosynthesis and gene expression of 30 out of $47 \mathrm{SM}$ gene clusters depend on availability of nitrogen (Wiemann et al., 2013; Tudzynski, 2014). Recently, we have shown that the global nitrogen regulators AreA and AreB play major roles in regulating nitrogen-controlled SM clusters (Michielse et al., 2014). Addition of preferred nitrogen sources, such as glutamine, to cultures led to rapid down-regulation of several nitrogen-repressed SM genes, e.g., the GA biosynthetic genes. By contrast, cultures supplied with nitrate showed a significant 
delay in repressing these clusters, probably due to the time-consuming conversion of nitrate to glutamine (Wagner et al., 2013). Although the ammonium permease (MepB) and the glutamine synthetase (GS) were shown to be involved in sensing ammonium and glutamine, respectively, nothing is known about potential nitrate sensors. Therefore, we wished to determine the molecular mechanisms involved in nitrate sensing, uptake, and gene regulation networks in F. fujikuroi.

The majority of fungi, with the exception of some yeast species, are able to assimilate nitrate or nitrite, the most abundant nitrogen ions present in soils and plants (Siverio, 2002; Song et al., 2007; Gorfer et al., 2011; Schinko et al., 2013). Utilization of nitrate requires its reduction to nitrite by the activity of nitrate reductase (NR) and reduction of nitrite to ammonium by nitrite reductase (NiR), respectively (Cove, 1979; Campbell and Kinghorn, 1990). This is an energy-consuming process and therefore, nitrate and nitrite are considered as unfavorable nitrogen sources that are only used when the preferred sources glutamine, glutamate, or ammonium are not available (Cove, 1979; Premakumar et al., 1979; Scazzocchio and Arst, 1989; Campbell and Kinghorn, 1990; Crawford and Arst, 1993; Siverio, 2002). It appears, therefore, that the nitrate assimilation system may be tightly regulated, as shown in many fungal species and plants. In Aspergillus nidulans, where the nitrate assimilation system has been thoroughly studied, the nitrate assimilation genes coding for NR (NIAD), NiR (NIIA), a nitrate/nitrite transporter (NRTA) and a specific nitrite transporter (NITA) are clustered, whilst a fifth gene, coding for a second transporter (NRTB) is located separately on the same chromosome (Cove, 1979; Brownlee and Arst, 1983; Johnstone et al., 1990; Unkles et al., 1991, 2001). These genes are only expressed when two conditions are met: low intracellular concentrations of preferred nitrogen sources (nitrogen-starvation conditions) and the simultaneous presence of nitrate or nitrite, which act as transcriptional inducers (reviewed by Marzluf, 1997). This expression pattern is subject to the synergistic action of the nitrate-specific regulator NirA (NIT4 in Neurospora crassa) and the global, nitrogen-dependent regulator AreA (NIT2 in N. crassa). In A. nidulans, the GAL4type binuclear $\mathrm{Zn}_{2} \mathrm{Cys}_{6}$ transcription factor (TF) NirA binds to non-palindromic consensus sequences ( $5^{\prime} \mathrm{CTCCGHGG3}{ }^{\prime}$ ) in the bidirectional promotor of NIAD and NIIA, mediating their transcription (Burger et al., 1991; Punt et al., 1995; Strauss et al., 1998). While the NIRA gene itself is constitutively expressed irrespective of the nitrogen conditions (Burger et al., 1991), the NirA protein is subject to a strict post-translational regulation by cellular translocation. The addition of nitrate, nitrite, or chlorate leads to a rapid nuclear accumulation of NirA, even in the simultaneous presence of preferred nitrogen sources such as ammonium (Berger et al., 2006). This nitrate-responsive nuclear shuttling is subject to the interaction of NirA with the nuclear export factor KapK. Recently, it was shown that nitrate leads to a conformational change of NirA, based on the redox-status of methionine 169 in the nuclear export sequence (NES) of the protein. Nitrate-induced reduction of Met169 presumably masks the NES, thus leading to a disrupted NirA-KapK interaction and nuclear retention of the protein
(Bernreiter et al., 2007; Gallmetzer et al., 2015). A similar nitrate-mediated nuclear shuttling was reported for the NirAhomolog NLP7 in the plant Arabidopsis thaliana (Marchive et al., 2013).

AreA, the second TF involved in control of the nitrate assimilation genes, belongs to the GATA family of $\mathrm{Cys}_{2}-\mathrm{Cys}_{2}$ TFs. In ascomycetes, it is considered as the major activator of genes involved in the utilization of alternative nitrogen sources, when preferred ones are absent. This kind of regulation, known as nitrogen metabolite repression (NMR), regulates not only components of the nitrate assimilation system, but also permeases and enzymes involved in the uptake and utilization of other nitrogen sources such as amino acids, GABA, or ammonium (Arst and Cove, 1973; Wiame et al., 1985; Marzluf, 1997; Magasanik and Kaiser, 2002; Macios et al., 2012; Michielse et al., 2014; Tudzynski, 2014; Pfannmüller et al., 2015). AreA itself is greatly repressed by high concentrations of nitrogen at the transcriptional, post-transcriptional, and posttranslational levels (Kudla et al., 1990; Platt et al., 1996; Tao and Marzluf, 1999; Morozov et al., 2000, 2001; Caddick et al., 2006).

In A. nidulans and N. crassa it was shown that AreA/NIT2 is essential for in vivo DNA-binding of NirA/NIT4 to promoters of nitrate assimilation genes and for their expression. AreA/NIT2 binds to the bi-directional NIIA-NIAD promoter in vivo under nitrogen-limiting conditions and at the same time, mediates promoter binding of NirA/NIT4 by direct protein-protein interaction (Feng and Marzluf, 1998; Narendja et al., 2002; Muro-Pastor et al., 2004; Berger et al., 2006). Furthermore, AreA indirectly affects nuclear translocation of NirA due to the AreA-dependent expression of nitrate transporters, which are needed for uptake of extracellular nitrate (Berger et al., 2006).

In addition to AreA, a second GATA-TF involved in nitrogendependent gene regulation has been described in fungi, known as AreB in Aspergillus and Fusarium species. While AreB was initially described as a negative counterpart of AreA that acts as a major repressor of AreA-activated nitrogen catabolism genes (Wong et al., 2009), recent studies have shown that its function is more complex (Haas et al., 1997; Dzikowska et al., 2003). Recently, we have shown that AreA and AreB can act as synergistic activators or repressors of their shared target genes, which includes the genes of the GA biosynthesis cluster (Michielse et al., 2014). No studies have been made of any possible impact of AreB on nitrate assimilation.

The transcriptional activation of the A. nidulans nitrate assimilation gene cluster is also controlled by chromatin remodeling. Under non-inducing conditions, six nucleosomes are positioned at the bidirectional NIIA-NIAD promotor. In the presence of nitrate, however, all nucleosomes lose positioning, giving way to an open chromatin structure that enables gene transcription. This process depends on AreAactivity and was the first example of a GATA factor that is involved in chromatin remodeling (Muro-Pastor et al., 1999; Berger et al., 2006, 2008). Under nitrogen starvation conditions, AreA binds to GATA sites in the promoter region, mediating histone $\mathrm{H} 3$ acetylation by possible recruitment of 
histone acetyltransferases (Todd et al., 2005; Berger et al., 2008). Additionally, the nitrate-induced TF NirA also influences the chromatin status, but only under nitrate induction conditions in an H3 acetylation-independent manner (Berger et al., 2008). AreA-dependent chromatin remodeling at the genomic locus of nitrate assimilation genes was also reported in Fusarium oxysporum (Malardier et al., 1989; López-Berges et al., 2010, 2014).

In this work, we studied the system of nitrate assimilation in F. fujikuroi, including its uptake, incorporation into metabolism and a possible direct sensing of nitrate and nitrite. We identified the main functional components involved in nitrate uptake and assimilation, including the genes encoding the $\mathrm{NR}, \mathrm{NiR}$, the main nitrate transporter NrtA and the TF NirA and studied their expression in response to the presence of nitrate, nitrite, and glutamine. We also analyzed the regulatory roles of the pathway-specific TF NirA and the global nitrogen-regulators AreA and AreB. Apart from transcriptional regulation, we investigated the regulation of the subcellular localization of the NirA::GFP fusion protein in the wild-type (Wt) and deletion mutants in response to different nitrogen sources.

\section{RESULTS}

\section{Identification of $F$ fujikuroi Nitrate Assimilation Genes}

To identify the respective orthologous nitrate assimilation genes, we performed a BLAST search on the F. fujikuroi genome database (Wiemann et al., 2013) with protein sequences of the NiR, the nitrate transporter NrtA and the TF NirA of $A$. nidulans and $F$. oxysporum as queries. The $F$. fujikuroi NIAD gene, coding for a putative NR, was previously identified (Tudzynski et al., 1996). For each sequence, one putative homolog with high sequence similarity was identified (Table 1) and the respective $F$. fujikuroi genes will be referred to as NIAD (FFUJ_12277, encoding NR), NIIA (FFUJ_06099, encoding NiR), NRTA (FFUJ_00934), and NIRA (FFFUJ_04567), respectively.

Notably, every single gene encoding one of the putative nitrate assimilation components is located on a different chromosome in F. fujikuroi (Table 1). This genomic arrangement is different from the one in A. nidulans, where NIAD, NIIA, and NRTA are located on the same chromosome in close proximity forming a co-regulated gene cluster (Cove, 1979; Brownlee and Arst, 1983; Johnstone et al., 1990). The scattering of the nitrate assimilation genes in the genome of $F$. fujikuroi may indicate a different, more uncoupled transcriptional regulation compared to $A$. nidulans.

The promotor sequences upstream of the NIRA, NIAD, NIIA, and NRTA genes were searched for putative binding sites of NirA (non-palindromic recognition sequence 5'CTCCGHGG3') and GATA-TFs like AreA (double GATA/TATC sequence elements with a distance of $<30 \mathrm{bp}$ ). The promoter sequences of NIAD, NIIA, and NRTA were found to contain at least one putative NirA-binding motif, while multiple double GATA-binding sites were present in the $5^{\prime}$ non-coding regions of all four genes (Table 1).

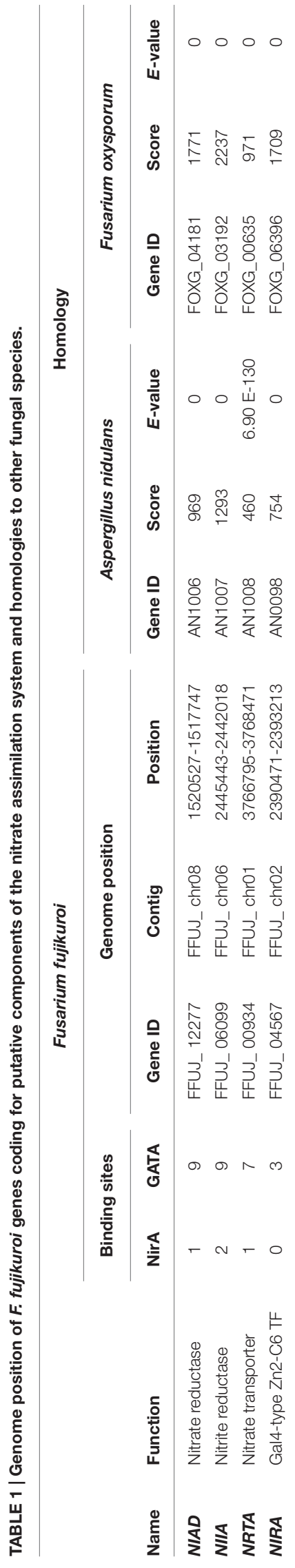




\section{NR, NiR, NrtA, and NirA Are the Main Components of Nitrate Assimilation in F. fujikuroi}

To elucidate the functions of the putative F. fujikuroi nitrate assimilatory genes, deletion mutants of NIIA, NRTA, and NIRA were generated. The F. fujikuroi Wt, the newly generated knockout strains and the already available $\triangle$ NIAD (Tudzynski et al., 1996), $\triangle A R E A$ (Tudzynski et al., 1999), and $\triangle A R E B$ (Michielse et al., 2014) deletion mutants were incubated for 4 days on solidified synthetic ICI minimal medium, supplemented with different nitrogen sources (Figure 1). All mutant strains were able to grow in a Wt-like manner on glutamine, with the exception of $\triangle A R E B$, which showed a slightly reduced growth. In contrast, only the Wt and $\triangle A R E B$ were able to grow with nitrate, clearly demonstrating that NR, NiR, NrtA, NirA, and AreA are crucial components for the use of nitrate as a nitrogen source. This was also reflected by a drastically reduced biomass formation of these mutants when cultivated in liquid cultures with nitrate as the sole nitrogen source (Supplementary Figure S1A).

On solid medium with nitrite, the colony diameter of the Wt was similar to that on glutamine and nitrate. However, the aerial mycelium appeared to be thinner, suggesting less biomass formation (Figure 1). This was confirmed by cultivation of the Wt in liquid medium with glutamine, nitrate or different concentrations of nitrite (Supplementary Figure S1B) and is probably caused by toxic effects of nitrite that inhibit growth of the fungus (Wodzinski et al., 1978; Crawford and Arst, 1993; Cabrera et al., 2014). The $\triangle N I A D$ and $\triangle N R T A$ mutants showed Wt-like growth on nitrite, whereas the $\triangle A R E B$ mutant grew with a slightly reduced colony diameter compared to the
Wt (Figure 1). In contrast, no growth on nitrite medium has been observed for the $\triangle N I I A, \triangle N I R A$, and $\triangle A R E A$ mutants, demonstrating that NiR is the only nitrite-reducing enzyme, and that the TFs NirA and AreA, but not AreB, are essential for the assimilation of nitrite (Figure 1). Furthermore, the Wt-like growth of $\triangle N R T A$ on nitrite showed that this transporter is essential for transport of nitrate, but not of nitrite (Figure 1).

We also studied the sensitivity of all strains to chlorate $\left(\mathrm{ClO}_{3}\right)$, a structural analog of nitrate. Chlorate is imported into the cell by nitrate transporters and acts as a substrate of the NR which reduces it to the highly reactive and toxic chlorite $\left(\mathrm{ClO}_{2}\right)$, thereby killing the cells (Cove, 1976; Brownlee and Arst, 1983; van Wijk et al., 1998). Accordingly, the F. fujikuroi Wt showed almost no growth on medium supplemented with chlorate due to the activity of the NR. The same was the case for the $\triangle$ NIIA, $\triangle$ NRTA, $\triangle N I R A$, and $\triangle A R E B$ mutants indicating that they are able to reduce chlorate to chlorite. However, deletion of NIAD and AREA mediated resistance to chlorate, probably due to the loss or drastic down-regulation of NR activity, respectively, in these mutants (Figure 1).

To elucidate the impact of AreA on the nitrate assimilation in more detail, we constitutively expressed the nitrate transporter gene NRTA in the background of the $\triangle A R E A$ deletion mutant. The resulting strain $\triangle A R E A-O E:: N R T A$ was unable to grow on nitrate and nitrite medium, but in contrast to the $\triangle A R E A$ mutant, was sensitive to chlorate due to the AreA-independent import of nitrate by the activity of the transporter NrtA and most likely low but sufficient activity of the NR in the $\triangle A R E A$ background.

Taken together, our growth analysis showed that NR, NiR, and NrtA are crucial components of the F. fujikuroi nitrate

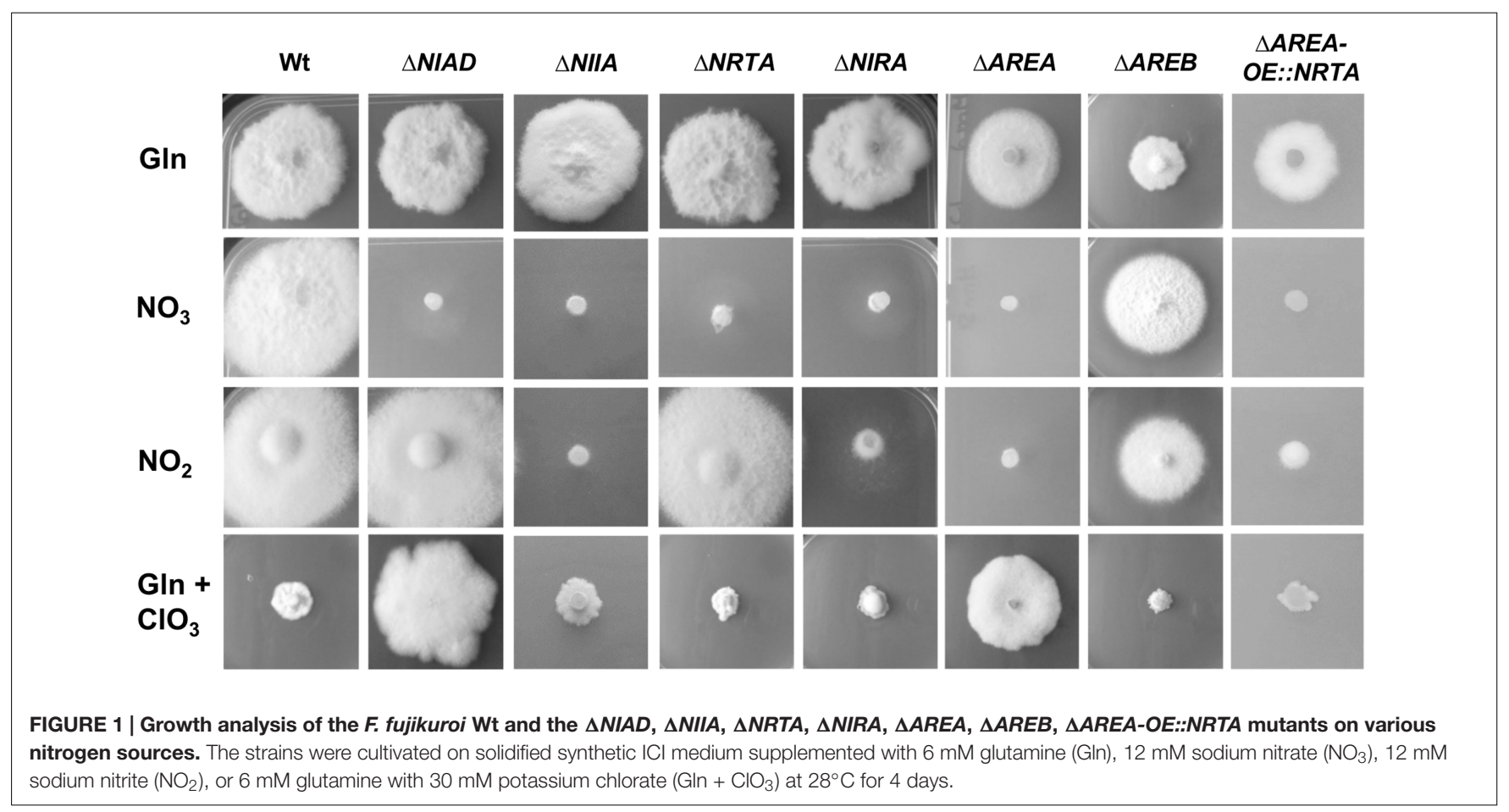


assimilation system as they are all needed for growth on nitrate, whereas NiR is essential for growth on nitrite. NrtA seems to be the major nitrate transporter of F. fujikuroi, but it is not or not solely involved in the uptake of nitrite. In addition, our plate assays clearly show that both the TF NirA and the global GATA-TF AreA are involved in the regulation of the nitrate and nitrite assimilation system.

\section{Is Nitrate Directly Sensed and Causes NMR?}

In F. fujikuroi, it was previously shown that the expression of many SM gene clusters is subject to NMR in an AreAdependent (e.g., GA biosynthesis genes) or AreA-independent [e.g., bikaverin (BIK) biosynthesis genes] manner (Candau et al., 1992; Mihlan et al., 2003; Wiemann et al., 2009, 2013; Tudzynski, 2014). The main effector mediating rapid and strong repression of these two SM clusters was shown to be glutamine, while addition of nitrate to cultures led to a significantly delayed down-regulation of these genes compared to glutamine (Wagner et al., 2013).

However, it is difficult to distinguish between a direct (though delayed) regulatory effect of nitrate itself and an indirect effect via its conversion to glutamine. Therefore, we investigated the different effects of nitrate and glutamine on the expression of GA and BIK genes during a time course of $2 \mathrm{~h}$ in the Wt and the $\triangle N I A D, \triangle N R T A, \triangle N I R A$, and $\triangle A R E A$ mutants. By using these mutants for expression analyses we were able to further differentiate between nitrate- and glutamine-mediated effects, because the uptake and/or the metabolization of nitrate and nitrite was clearly abolished in these mutants (Figure 1). Since the deletion mutants are unable to grow with nitrate as the sole nitrogen source, we cultivated the F. fujikuroi Wt and the $\triangle N I A D, \triangle N R T A, \triangle N I R A$, and $\triangle A R E A$ mutants initially in synthetic medium with $6 \mathrm{mM}$ glutamine for 3 days. At this time $(72 \mathrm{~h})$, glutamine was depleted and the fungus coped with nitrogen-starvation $(-\mathrm{N})$ as indicated by high expression of GA and BIK biosynthetic genes (Figure 2). The cultures were then supplemented with either $60 \mathrm{mM}$ glutamine or $60 \mathrm{mM}$ nitrate, and mycelia were harvested $0.5,1$, or $2 \mathrm{~h}$ after the addition of nitrogen to study the expression of the GA and BIK biosynthetic genes, $C P S / K S$ and $B I K 2$, respectively, by Northern blot analysis (Figure 2).

Under nitrogen-starvation (after $72 \mathrm{~h}$ growth in $6 \mathrm{mM}$ glutamine), $C P S / K S$ and $B I K 2$ were highly expressed in the $\mathrm{Wt}$ and all deletion mutants, except for $C P S / K S$ in the $\triangle A R E A$ mutant due to the AreA-dependent expression of GA genes (Mihlan et al., 2003). The addition of high concentrations of glutamine resulted in rapid and total loss of $C P S / K S$ expression in all strains, while BIK2 expression was detected in the Wt up to $1 \mathrm{~h}$ after glutamine addition. Surprisingly, a de-regulation of $B I K$ expression was observed in $\triangle N I R A, \triangle N I A D$, and $\triangle N R T A$ after addition of glutamine, because expression was clearly detected in these mutants up to $2 \mathrm{~h}$ in contrast to the $\mathrm{Wt}$.

When high concentrations of nitrate were added, transcripts of both $C P S / K S$ and $B I K$ were clearly visible in the Wt up to $2 \mathrm{~h}$ after nitrate addition, though with decreasing intensity
(Figure 2). This time-delay of gene repression in response to nitrate could be an indication that nitrate is not directly sensed as an effector of NMR, and that down-regulation of genes began only after its conversion into glutamine, the real effector of NMR. This hypothesis is further supported by the fact that no obvious repression was observed up to $2 \mathrm{~h}$ after nitrate-addition in $\triangle N I R A, \triangle N I A D$, and $\triangle N R T A$ mutants. These mutants were unable to utilize nitrate, and therefore no accumulation of the main NMR-effector glutamine could take place.

In summary, our expression analysis revealed that the genes of the GA and BIK cluster are quickly repressed by glutamine, but are not directly affected by nitrate. Instead, the delayed repression in comparison to that for glutamine is due to the time-consuming conversion of nitrate to glutamine. In contrast to the Wt, we did not observe a significant repression of GA and BIK genes in the $\triangle N I R A, \triangle N I A D$, and $\triangle N R T A$ mutants in response to nitrate as all these mutants are unable to metabolize nitrate. The data indicated that nitrate is not sensed directly as an effector of NMR in F. fujikuroi.

\section{Transcriptional Regulation of Nitrate Assimilatory Genes}

In $A$. nidulans, the expression of the co-regulated nitrate assimilation genes NIAD, NIIA, and NRTA is induced by nitrate and nitrite and depends on the activity of both the specific TF NirA and the global, nitrogen-dependent regulator AreA (Berger et al., 2006). To study the transcriptional regulation of these genes in F. fujikuroi, the Wt and the $\triangle N I A D, \triangle N R T A$, $\triangle N I R A$, and $\triangle A R E A$ mutants were first cultivated for 3 days with $6 \mathrm{mM}$ glutamine (nitrogen-limiting conditions) before high concentrations of either $60 \mathrm{mM}$ glutamine or $60 \mathrm{mM}$ nitrate were added to the cultures. Expression of the genes NIRA, NIAD, and NRTA was studied before, and 0.5 or $2 \mathrm{~h}$ after the addition of nitrogen (Figure 3A).

A weak or no expression of the TF-encoding gene NIRA was detected in the Wt under nitrogen-starvation conditions and after the addition of glutamine, respectively, indicating that NIRA is subject to NMR. After addition of nitrate, NIRA transcripts were detected at $0.5 \mathrm{~h}$, but were completely absent after $2 \mathrm{~h}$, likely due to the accumulation of glutamine similar to the delayed nitrate-repression of the GA and BIK cluster genes (Figure 2). Accordingly, NirA expression was still detected $2 \mathrm{~h}$ after nitrate addition in the $\triangle N I A D$ and $\triangle A R E A$ mutants, which are unable to convert nitrate to glutamine (Figure 3A). Surprisingly, the expression levels of NIRA were significantly elevated in $\triangle A R E A$ compared to the Wt under all tested conditions, indicating a repressive impact of AreA on NIRA expression.

Expression of NIAD and NRTA was not detected in any strain under nitrogen-starvation conditions or after addition of glutamine (Figure 3A). However, $0.5 \mathrm{~h}$ after addition of nitrate, strong expression of both genes was detected in the $\mathrm{Wt}$, but was significantly reduced or abolished at $2 \mathrm{~h}$. It was notable that the same nitrate-dependent induction of NIAD-expression was detected in the $\triangle N R T A$ mutant - which, based on our plate assays, was hitherto assumed to be unable to import nitrate into the cell (Figure 1). Furthermore, a strong expression of NRTA 


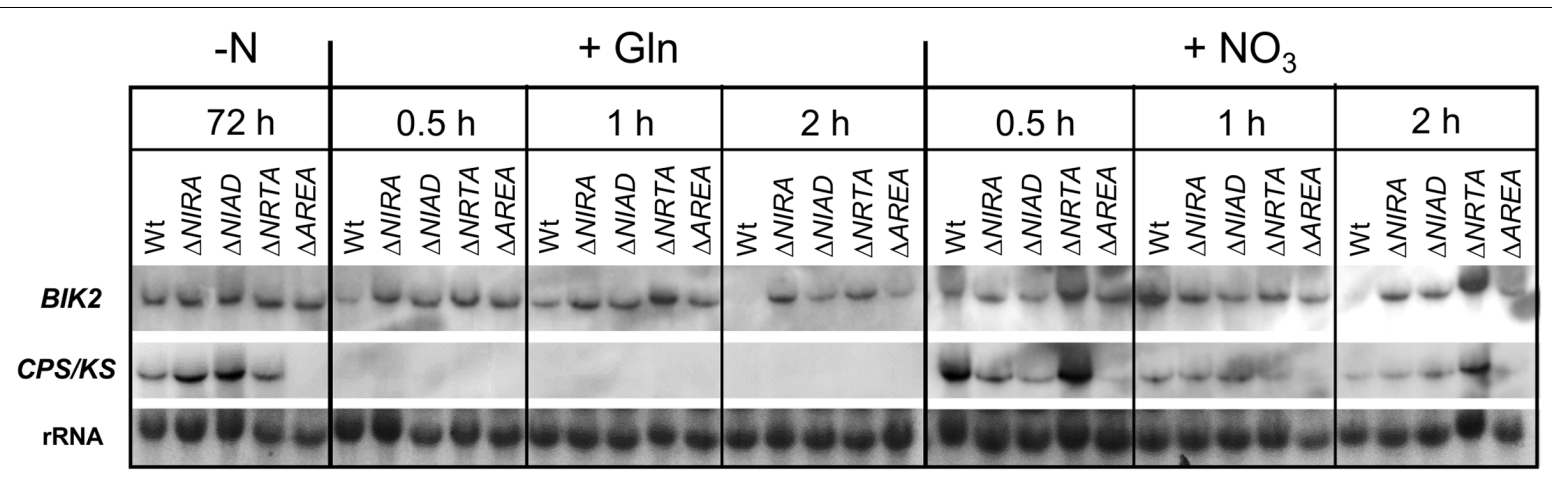

FIGURE 2 | Time-course of GA and BIK biosynthetic gene expression in response to high concentrations of glutamine and nitrate. The $F$. fujikuroi Wt and the $\triangle$ NIRA, $\triangle$ NIAD, $\triangle$ NRTA, $\triangle$ AREA mutants were cultivated in liquid synthetic $\mathrm{ICl}$ medium at $28^{\circ} \mathrm{C}$ for 3 days ( $-\mathrm{N}$ ). Cultures were then supplemented with $60 \mathrm{mM}$ glutamine (+Gln) or $60 \mathrm{mM}$ sodium nitrate $\left(+\mathrm{NO}_{3}\right)$. Mycelia were harvested before $(-\mathrm{N})$ or $0.5,1$, or $2 \mathrm{~h}$ after the addition of the respective nitrogen sources and expression of CPS/KS (copalyl diphosphate/kaurene-synthase, GA-cluster) and BIK2 (monooxygenase, BIK-cluster) was analyzed by Northern blot.

was observed in the $\triangle N I A D$ mutant under nitrogen-limiting conditions without nitrate. However, the addition of glutamine immediately resulted in NRTA-repression. After addition of nitrate, the expression was still high at $2 \mathrm{~h}$ when the Wt no longer showed NRTA expression, probably due to the unmetabolized presence of nitrate in this mutant (Figure 3A).

In a second experiment, we compared the effects of nitrate and nitrite on the expression of NIAD and NIIA. Therefore, the F. fujikuroi Wt, the $\triangle N I A D, \triangle N I I A, \triangle N R T A, \triangle N I R A$, and $\triangle A R E A$ mutants were cultivated for 3 days as described above and then supplemented with $60 \mathrm{mM}$ glutamine, $12 \mathrm{mM}$ nitrate, or $12 \mathrm{mM}$ nitrite for $0.5 \mathrm{~h}$ each (Figure 3B). NIAD and NIIA expression followed the same nitrate-induced pattern as in the previous experiment (Figures 3A,B). In contrast, no expression of NIAD or NIIA was observed upon the addition of nitrite, the substrate of NiR (Figure 3B). In a similar manner to NRTA, NIIA was strongly expressed in the $\triangle$ NIAD mutant under nitrogen-starvation conditions without the addition of nitrate (Figure 3B).

The expression pattern of the nitrate assimilation genes in the background of the $\triangle N I R A$ and $\triangle A R E A$ deletion mutants provide insights to the possible regulatory role of the two TFs (Figure 3). In $\triangle N I R A$, no expression was observed for NIAD, NIIA, and NRTA under all tested conditions, clearly showing that NirA acts as an obligate and specific transcriptional activator for these genes. Similarly, no expression of NIIA and NRTA was observed in $\triangle A R E A$. A weak, but clearly visible, expression of NIAD was detected in $\triangle A R E A$ mutant after addition of nitrate indicating that AreA probably acts as a strong, though not an obligate transcriptional activator of NIAD.

Taken together, our expression analysis revealed that the main nitrate assimilation genes NIAD, NIIA, and NRTA are repressed by high concentrations of glutamine and highly expressed upon induction with nitrate, but not nitrite. The TFs NirA and AreA are both needed for transcriptional activation of NIIA and NRTA, and NirA for transcription of NIAD. AreA acts also as a strong positive regulator of NIAD, but is not essential for its expression.

\section{NirA Is Regulated by Nucleocytoplasmatic Shuttling}

Apart from a nitrate-dependent regulation at the transcriptional level, the pathway-specific TF NirA was shown to be regulated on a post-translational level in A. nidulans, by a nitratedependent shuttling from a primary cytosolic localization to an accumulation inside the nucleus (Berger et al., 2006). To investigate a similar localization-dependent regulation of NirA in F. fujikuroi, a NirA::GFP fusion construct was constitutively expressed in the $\triangle N I R A$ mutant, resulting in strain $\triangle N I R A$ NIRA::GFP. A plate assay showed that the fusion construct was functional because the growth defect shown by $\triangle N I R A$ was fully restored on nitrate and nitrite medium by expression of NirA::GFP (Supplementary Figure S2).

For microscopic observation, the $\triangle N I R A-N I R A:$ GFP strain and the Wt (as a control) were cultivated in liquid ICI medium with $6 \mathrm{mM}$ glutamine until the cells were starved for nitrogen. The $\triangle N I R A-N I R A:: G F P$ cultures were then supplemented with either $6 \mathrm{mM}$ glutamine, $12 \mathrm{mM}$ nitrate, or $12 \mathrm{mM}$ nitrite, and the cellular localization of NirA::GFP was examined immediately after $(<30 \mathrm{~s})$ by fluorescence microscopy. Cells were simultaneously stained with Hoechst solution for visualization of nuclei (Figure 4). With the glutamine supplement, the $\triangle N I R A-N I R A:: G F P$ cells showed a very faint fluorescence inside round structures, overlapping with the Hoechst staining of nuclei (Figure 4B). However, the same faint nuclear signals were detected in the $\mathrm{Wt}$, indicating that it is not a specific GFP signal, but instead a non-specific background fluorescence caused by the Hoechst solution as seen in the GFP filter set (Figure 4A). Additionally, we never observed a similar nuclear GFP signal with glutamine without simultaneous Hoechst staining. Therefore, the NirA::GFP fusion protein does not appear to be localized to particular structures under glutamine conditions and is most likely distributed evenly inside the cell. Similarly, no specific localization of the fusion protein was observed after the addition of nitrite (Figure 4B). In contrast, a strong fluorescence signal was detected inside the nucleus of the 


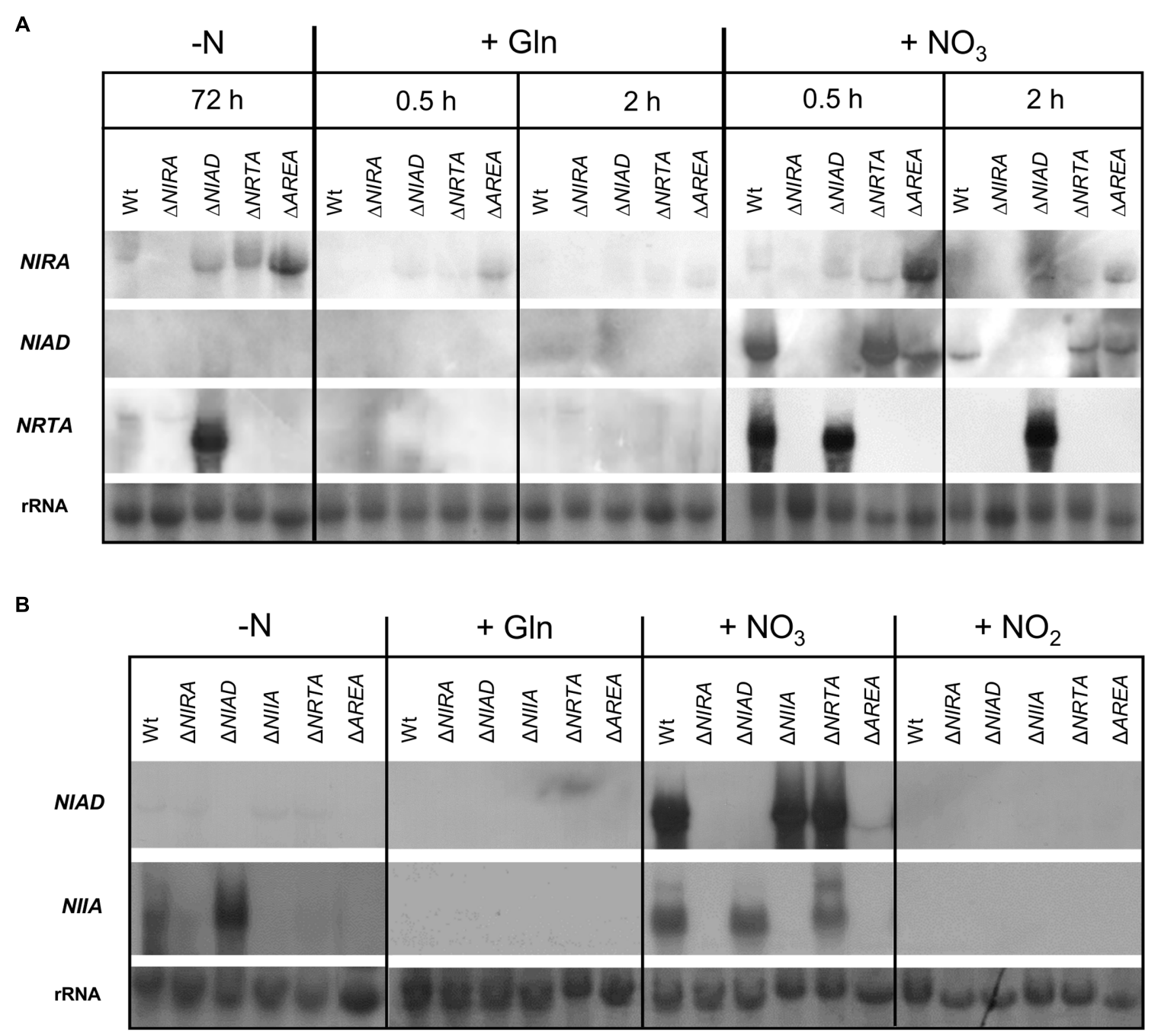

FIGURE 3 | Expression of nitrate assimilation genes in response to different nitrogen sources. The $F$. fujikuroi Wt and the $\triangle N I R A, \triangle N I A D, \triangle N R T A, \triangle A R E A$ mutants were cultivated in liquid synthetic $\mathrm{ICl}$ medium at $28^{\circ} \mathrm{C}$ for 3 days $(-\mathrm{N})$. (A) Cultures were supplemented with $60 \mathrm{mM}$ glutamine (+GIn) or $60 \mathrm{mM}$ sodium nitrate $\left(+\mathrm{NO}_{3}\right)$. Mycelia were harvested before $(-\mathrm{N})$ or $0.5 \mathrm{~h}$ or $2 \mathrm{~h}$ after the addition of the respective nitrogen sources and expression of $\mathrm{NIRA}$, NIAD, and NRTA was analyzed by Northern blot. (B) Cultures were supplemented with $30 \mathrm{mM}$ glutamine (+Gln), $12 \mathrm{mM}$ sodium nitrate (+NO 3$)$ or $12 \mathrm{mM}$ sodium nitrite (+NO 2$). \mathrm{Mycelia}$ were harvested before $(-\mathrm{N})$ or $0.5 \mathrm{~h}$ after the addition of the respective nitrogen sources and expression of NIAD and NIIA was analyzed by Northern blot.

$\triangle N I R A-N I R A:: G F P$ strain immediately after addition of nitrate (Figure 4B).

To assess the total protein level of NirA::GFP under different nitrogen conditions, we performed an immunoblot analysis. Nitrogen-starved cultures of $\triangle N I R A-N I R A:: G F P$ were supplemented with $12 \mathrm{mM}$ nitrate, $12 \mathrm{mM}$ nitrite, or $60 \mathrm{mM}$ glutamine. Cells were harvested before the addition of nitrogen, and 5 and $60 \mathrm{~min}$ after addition of nitrogen, and the total protein was extracted for Western blot analysis (Figure 5A). The fusion protein was neither detected before, nor 5 min after addition of nitrate, and only a faint signal was visible 5 and 60 min after addition of nitrite or glutamine. In contrast, a strong signal of NirA::GFP was detected 60 min after addition of nitrate demonstrating that only nitrate, but not nitrite or glutamine, causes a significant accumulation of the NirA protein
(Figure 5A). It should be noted that this accumulation of the NirA::GFP protein took between 5 and $60 \mathrm{~min}$, whereas the strong nuclear accumulation was completed significantly faster in a time-span of less than $30 \mathrm{~s}$ (Figure 4A).

In order to investigate a possible impact of the other components of the nitrate assimilation system on NirAlocalization, we also expressed the NirA::GFP fusion protein in the background of the $\triangle N R T A, \triangle A R E A$, and $\triangle N I A D$ deletion mutants. The resulting strains $\triangle N R T A-N I R A:: G F P, \triangle A R E A-$ NIRA::GFP, and $\triangle N I A D-N I R A:: G F P$, as well as strain $\triangle N I R A-$ NIRA::GFP (control) were cultivated as described above, after which $6 \mathrm{mM}$ glutamine or two different concentrations of nitrate $(12 \mathrm{mM}$ or $1 \mathrm{mM})$ were added. The cells were then examined immediately afterward by fluorescence microscopy (Figure 6). 
A

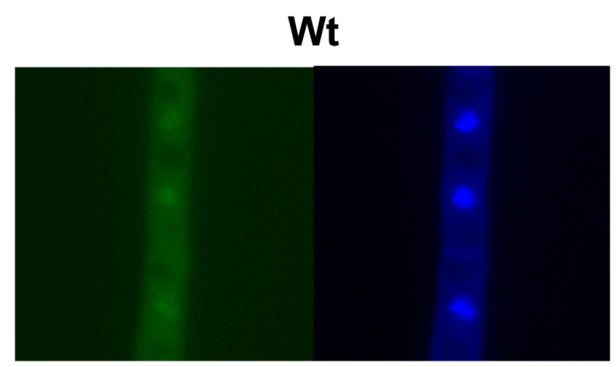

B

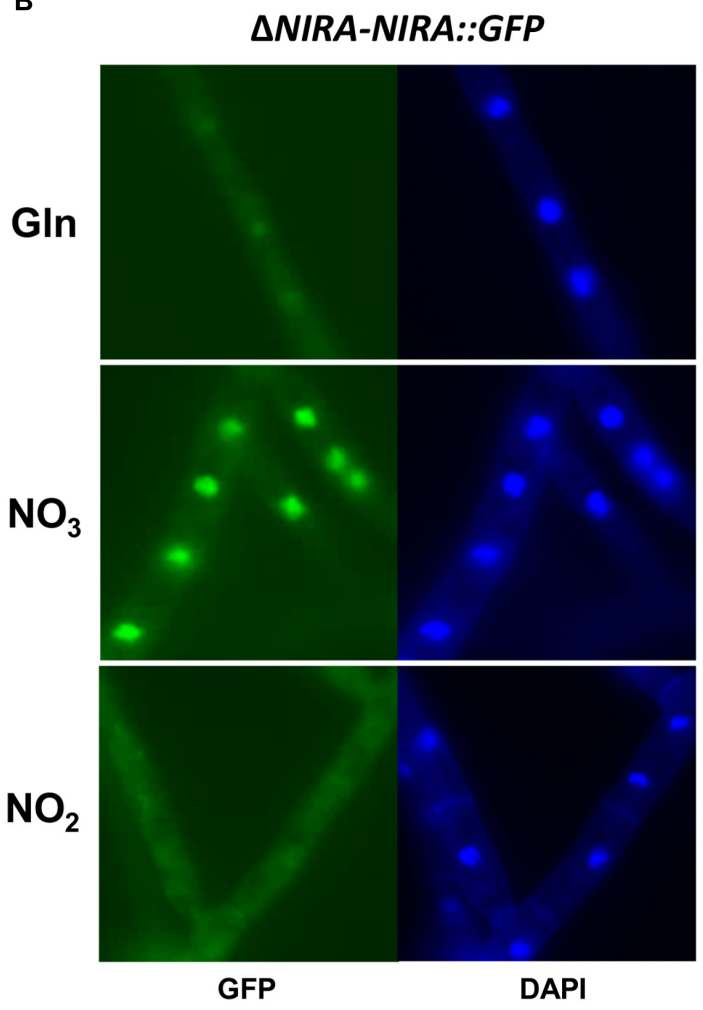

FIGURE 4 | Nitrate-dependent nuclear translocation of the NirA::GFP fusion protein. The $F$. fujikuroi $\mathrm{Wt}$ (A) and the $\triangle N I R A$ mutant transformed with a constitutively expressed NirA::GFP fusion construct (B) was cultivated in liquid synthetic $\mathrm{ICl}$ medium with $6 \mathrm{mM}$ glutamine for 2 days at $28^{\circ} \mathrm{C}$. Cells were observed by fluorescence microscopy (GFP) less than 1 min after the addition of $6 \mathrm{mM}$ glutamine (Gln), $12 \mathrm{mM}$ sodium nitrate $\left(\mathrm{NO}_{3}\right)$, or $12 \mathrm{mM}$ sodium nitrite $\left(\mathrm{NO}_{2}\right)$. Additionally, cells were stained with Hoechst solution for visualization of nuclei (DAPI).

We observed a similar nitrate-induced nuclear accumulation of NirA::GFP as in our previous experiment (Figure 4), even with the lowest concentration of nitrate (1 mM) (Figure 6). The same localization pattern of NirA::GFP was also observed in the $\triangle N R T A$ and $\triangle A R E A$ backgrounds suggesting that small amounts of nitrate are present in the cells. In contrast, no nuclear accumulation of NirA::GFP in response to nitrate was observed in the $\triangle N I A D$ background. We observed only a faint, evenly distributed GFP fluorescence in the cytosol, indicating that the NR appears to be involved in regulating the nitrate-dependent NirA-localization.



B

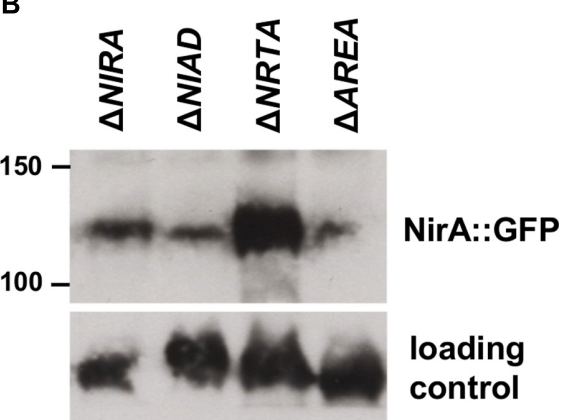

FIGURE 5 | Abundance of NirA::GFP protein after exposure to different nitrogen sources. (A) The F. fujikuroi $\triangle N I R A$ mutant transformed with a constitutively expressed NirA::GFP fusion construct was cultivated in liquid synthetic $\mathrm{ICl}$ medium with $6 \mathrm{mM}$ glutamine for 3 days at $28^{\circ} \mathrm{C}$. Cultures were harvested before $(-\mathrm{N})$ and $5 \mathrm{~min}$ as well as $60 \mathrm{~min}$ after addition of $12 \mathrm{mM}$ sodium nitrate $\left(+\mathrm{NO}_{3}\right), 12 \mathrm{mM}$ sodium nitrite $\left(+\mathrm{NO}_{2}\right)$ and $60 \mathrm{mM}$ glutamine (+ Gln). (B) The F. fujikuroi $\triangle$ NIRA, $\triangle$ NIAD, $\triangle$ NRTA, and $\triangle A R E A$ mutants transformed with a constitutively expressed NirA::GFP fusion construct was cultivated in liquid synthetic $\mathrm{ICI}$ medium with $6 \mathrm{mM}$ glutamine for 3 days at $28^{\circ} \mathrm{C}$. Cultures were harvested $60 \mathrm{~min}$ after addition of $12 \mathrm{mM}$ sodium nitrate. Total protein was extracted and analyzed by Western blot using polyclonal anti-GFP antibodies for detection of NirA::GFP. Hybridization with anti- $\beta$-actin antibodies was used as protein loading control. Molecular weights are indicated in $\mathrm{kDa}$.

To address the possibility that the NirA::GFP protein is degraded in the $\triangle N I A D$ mutant, in contrast to the other mutants, the fusion protein was assessed 60 min after addition of nitrate in the different deletion backgrounds by Western blot analysis (Figure 5B). Since the fusion protein was ectopically expressed in all strains, the basal expression level might differ depending on the number of genomic integrations and the integration loci. Therefore, a direct quantitative comparison of the NirA::GFPlevels in $\triangle N I R A, \triangle N I A D, \triangle N R T A$, and $\triangle A R E A$ was not possible. However, the fusion protein was clearly detected in all four strains under nitrate conditions (Figure 5B), proving that the lack of nuclear NirA::GFP localization in $\triangle N I A D$ is the result of a disrupted nucleocytoplasmic shuttling and not an indirect effect caused by absence of the fusion protein.

Taken together, microscopic observations of the subcellular localization of the NirA::GFP fusion protein revealed that the TF is regulated on a post-translational level by nitrate- but not nitrite-dependent fast nuclear translocation and slower de novo NirA protein synthesis and potentially nitrate-induced protein 



GFP

BF

GFP



BF

FIGURE 6 | Involvement of NrtA, AreA, and NiaD in nitrate-dependent nuclear translocation of the NirA::GFP fusion protein. The F. fujikuroi $\triangle$ NIRA, $\triangle$ NRTA, $\triangle A R E A$, and $\triangle$ NIAD mutants transformed with a constitutively expressed NirA::GFP fusion construct were cultivated in liquid synthetic ICl medium with $6 \mathrm{mM}$ glutamine for 2 days at $28^{\circ} \mathrm{C}$. Cells were observed by fluorescence (GFP) and brightfield (BF) microscopy after the addition of $6 \mathrm{mM}$ glutamine (Gln), 12 mM sodium nitrate $\left(12 \mathrm{mM} \mathrm{NO}_{3}\right)$, or $1 \mathrm{mM}$ sodium nitrate $\left(1 \mathrm{mM} \mathrm{NO}_{3}\right)$.

stabilization. Evidence suggests that the NirA-translocation apparently depends on the NR, while the nitrate transporter NrtA and the GATA-TF AreA are not involved.

\section{DISCUSSION}

In this work, we studied the molecular mechanisms of the nitrate assimilation system in F. fujikuroi. We identified and functionally characterized the main genes involved in nitrate and nitrite assimilation and investigated the impact of the pathway-specific TF NirA and the global nitrogen regulator AreA on their expression.

\section{NR and NiR Are the Sole Nitrate and Nitrite Reductases, Respectively, in F. fujikuroi}

Our sequence analysis revealed that the genes NIAD and NIIA are the only genes in the genome of $F$. fujikuroi that encode NR and NiR, respectively. Deletion of the two genes confirmed their putative functions: $\triangle N I A D$ was no longer able to use nitrate, while $\triangle N I I A$ was unable to use nitrate or nitrite. Additionally, deletion of NIAD mediated resistance to chlorate (Figure 1). The growth analysis also clearly showed that the loss of NR or NiR activity by deleting NIAD or NIIA, respectively, cannot be compensated by any other enzyme. This proves that NIAD encodes the sole functional NR and NIIA encodes the sole functional NiR of F. fujikuroi.

\section{Nitrate-Uptake Depends on the Transporter NrtA}

In contrast to $A$. nidulans, where two functional nitrate transporters have been described and characterized (Brownlee and Arst, 1983; Unkles et al., 1991, 2001), we identified only a homolog of one of the A. nidulans nitrate transporters, NrtA. This is similar to F. oxysporum, where only a single nitrate transporter homolog is also present (López-Berges et al., 2014).

The generated F. fujikuroi $\triangle N R T A$ mutant was not able to grow on nitrate medium, clearly showing that NrtA is the major nitrate transporter (Figure 1). However, NrtA cannot be the only nitrate/chlorate transporter, because the deletion of NRTA did not mediate resistance to chlorate. In addition, nitrate-induced nuclear translocation of NirA::GFP in the $\triangle N R T A$ background suggested that at least small amounts of nitrate can be transported into the cell by alternative transporters. Furthermore, the Wtlike growth of $\triangle N R T A$ with nitrite as the sole nitrogen-source demonstrated that nitrite is internalized by other transporters. However, it cannot be excluded that NrtA is also able to transport nitrite.

A distinct transporter for uptake of nitrite named NitA/Nrt2, has already been described in A. nidulans (Wang et al., 2008) and F. oxysporum (López-Berges et al., 2014), respectively. A BLAST 
analysis of the F. fujikuroi genome revealed the presence of a putative nitrite transporter (FFUJ_03993) with significant homologies to NitA/Nrt2 (data not shown). Furthermore, the studies in A. nidulans showed that NitA is a specific transporter for the uptake of nitrite, while the two nitrate transporters NrtA and NrtB are able to additionally transport nitrite (Wang et al., 2008). A similar dual role as a nitrate and nitrite transporter may also be the case for NrtA in F. fujikuroi and should be investigated further in the course of characterizing the putative nitrite transporter.

\section{Expression of Nitrate Assimilation Genes Depends on Nitrate and the Regulator NirA, But Not Completely on AreA}

As in other fungi (Cove, 1979; Dunn-Coleman et al., 1981; Crawford and Arst, 1993; Marzluf, 1997; Siverio, 2002), the expression of the nitrate assimilation genes NIAD, NIIA, and NRTA was induced by the addition of nitrate to the growth medium and repressed by high concentrations of glutamine (Figure 3). An induction of NIIA and NRTA gene expression was also observed in the NIAD deletion mutant without the addition of nitrate, most likely due to trace amounts of nitrate that accumulate in this strain over time. A similar effect on gene expression was also described for the $A$. nidulans $\triangle$ NIAD mutant and termed as 'pseudo-constitutive' expression (Schinko et al., 2013).

Additionally, the TF NirA apparently acts as an obligate transcriptional activator of all $F$. fujikuroi nitrate assimilation genes, as no expression of NIAD, NIIA, and NRTA was observed in the $\triangle N I R A$ mutant. We identified potential NirA-binding motifs in the promotor sequences of all three genes, suggesting that NirA most likely activates their expression by directly binding to the respective promoters. A similar transcriptional activation of nitrate assimilation genes by promoter-binding of NirA/Nit4 has been described in A. nidulans and N. crassa (Burger et al., 1991; Yuan et al., 1991; Punt et al., 1995; Feng and Marzluf, 1998; Strauss et al., 1998).

Accordingly, the NIRA mutant is unable to grow on nitrate or nitrite, indicating that the NrtA, NR, and NiR activities must be drastically reduced in this mutant. However, despite the obligate dependency of the nitrate assimilation on NirA, the $\triangle N I R A$ mutant was unable to grow on medium supplemented with the toxic nitrate analog chlorate, indicating that at least low NR activity must be retained. This low activity is most likely not sufficient to support the growth on nitrate. This becomes apparent when compared to the $\triangle N I A D$ mutant, which displayed full chlorate resistance due to total loss of NR activity (Figure 1).

In $A$. nidulans and $N$. crassa it has been shown that the GATA-TF AreA/Nit2 is essential for the promoter-binding of NirA/Nit4 which involves direct protein-protein interaction (Arst and Cove, 1973; Feng and Marzluf, 1998; Muro-Pastor et al., 1999; Narendja et al., 2002; Berger et al., 2006). Furthermore, it has been shown that AreA is involved in opening the chromatin structure at the genomic loci of the nitrate assimilation genes in A. nidulans and F. oxysporum (Muro-Pastor et al., 1999; Todd et al., 2005; Berger et al., 2008; López-Berges et al., 2010, 2014).
Our results indicated that AreA acts as a strong positive regulator of the nitrate assimilation genes in F. fujikuroi: the $\triangle A R E A$ mutant was not able to grow on nitrate and nitrite, but in turn was resistant to chlorate (Figure 1). Consequently, no expression of NRTA or NIIA was observed upon deletion of AREA. Surprisingly, the expression of NIAD was only down-regulated in $\triangle A R E A$, but not totally abolished. As the NIAD transcription does not completely depend on AreA, the aforementioned chlorate resistance of the $\triangle A R E A$ mutant is most likely caused by the completely blocked import of chlorate due to the down-regulation of NRTA and additional uncharacterized transporters with non-specific transport activity for nitrate/chlorate by the global TF AreA. The constitutive, AreA-independent, expression of the main nitrate/chlorate transporter NrtA in the $\triangle A R E A$ background (Figure 1) did not restore growth on nitrate medium but restored the susceptibility to chlorate. These data clearly demonstrate that, in accordance with our expression analysis, a low NR activity must be left in $\triangle A R E A$ which is insufficient for growth on nitrate but adequate for conversion of chlorate to toxic levels of chlorite. The presence of additional transporters with low affinity for nitrate/chlorate besides NrtA would explain why the deletion of NRTA alone did not mediate chlorate resistance.

The partial down-regulation of NIAD expression in the $\triangle A R E A$ mutant can be seen in sharp contrast to $A$. nidulans where AreA-homologs were described as obligate activators of all nitrate assimilation genes (Muro-Pastor et al., 1999; Narendja et al., 2002; Berger et al., 2006, 2008). This difference in AreAdependent regulation between the fungal species is intriguing and is most likely reflected by the genomic localization of the nitrate assimilation genes in the different fungal species. While NIAD, NIIA, and NRTA are clustered in A. nidulans (Schinko et al., 2013), they are scattered across different chromosomes in F. fujikuroi. It is possible that clustering of genes makes them more accessible to histone rearrangements along the whole genomic region. A possible involvement of AreA in regulating the chromatin accessibility of nitrate assimilation genes in F. fujikuroi should be investigated in future studies to elucidate the potential differences in the regulatory mechanisms between the fungal species.

\section{NirA Is Sorted to the Nucleus in a Nitrate- and NR-Dependent Manner}

The gene encoding the pathway-specific TF NirA is weakly expressed under nitrogen-limiting conditions, irrespective of the presence of nitrate in F. fujikuroi (Figure 3A), similar to the situation in $A$. nidulans (Burger et al., 1991). In addition to transcriptional regulation, NirA in A. nidulans was shown to be regulated by a nitrate-, nitrite-, and chlorate-induced nuclear translocation (Berger et al., 2006; Schinko et al., 2013).

Our results suggest a similar regulation of NirA by nucleocytoplasmatic shuttling in F. fujikuroi. Under low glutamine conditions, the protein was mainly localized in the cytoplasm due to basal constitutive expression levels of the NIRA gene. Upon the availability of nitrate, but surprisingly not nitrite, NirA quickly accumulates in the nucleus (Figure 4). We 
suggest that the nuclear translocation shown to occur within mere seconds allows the immediate transcriptional activation of the main nitrate assimilation genes and a rapid adaption to changing nitrogen conditions. A regulation of NirA abundance via de novo protein synthesis would take longer (minutes) due to the time-consuming process of gene transcription and translation (Aymoz et al., 2016).

Our localization studies demonstrated that the nitrate-induced translocation of NirA is independent of the TF AreA and the nitrate transporter NrtA. In contrast, the nuclear accumulation of NirA was significantly less efficient upon addition of nitrate in the AREA deletion background in A. nidulans, most likely due to the lack of AreA-dependent nitrate transporter activation and the resulting limitation of the nitrate influx (Wang et al., 2008).

Beside the regulation via subcellular shuttling, regulation of the nitrate assimilation pathway is also mediated at the level of transcript stability in A. nidulans. For a number of genes, e.g., for NIAD and NIIA, destabilization of the transcripts in response to glutamine and stabilization of the transcripts in response to nitrate has been demonstrated, which depends on the $3^{\prime}$ UTR of the respective gene (Caddick et al., 2006). However, this mechanism cannot be responsible for the specific accumulation of NirA we observed 60 min after addition of nitrate (Figure 5) because the NIRA::GFP fusion construct is not terminated by the native NIRA terminator but instead by the heterologous glucoamylase terminator of $A$. nidulans

Therefore, the strong accumulation of the NirA protein in a time course of $60 \mathrm{~min}$ is likely due to nitrate-induced protein stabilization, e.g., by regulating proteasome-mediated degradation of target proteins via ubiquitination and stabilization through de-ubiquitination (Leach and Brown, 2012). However, the observed quick nuclear accumulation of NirA almost immediately after nitrate addition cannot be explained by de novo protein synthesis due to the time-delay between both effects. It is most likely that small amounts of NirA are always present inside the cell (due to the constitutive expression of the NIRA locus, Figure 3), which are immediately translocated to the nucleus in a short-term response to nitrate. As a second effect, the presence of nitrate induces an overall stabilization of the NirA protein, leading to its accumulation over time and enhancement of its activity. This multi-level regulation of NirA would enable the fungus to quickly adapt to the new conditions and to differentiate between a short- and long-term response to nitrate.

One of the most striking results of our work is the potential role of the NR in nitrate sensing. In contrast to A. nidulans, where a 'pseudo-constitutive' nuclear localization of NirA was observed in the $\triangle$ NIAD mutant (Schinko et al., 2013), we did not observe any detectable nuclear accumulation of NirA in the NIAD deletion background (Figure 6), which is not caused by a general lack of the protein in this strain (Figure 5B). This suggests that the NR is able to sense its nitrate substrate and transduce the nitrate signal to NirA. However, despite the fact that we did not see a clear nuclear localization of NirA in the $\triangle$ NIAD mutant, at least small amounts of NirA must be present inside the nucleus because the expression of the NirA-activated genes in response to nitrate was unaffected in this mutant (Figure 3).

\section{Differences between Nitrate- and Nitrite-Induced Responses}

In contrast to nitrate, we neither observed any expression of nitrate/nitrite assimilation genes (Figure 3B), nor increased protein levels (Figure 5) and nuclear translocation of NirA (Figure 4) in response to nitrite. This is surprising, because nitrite, in a similar manner to nitrate and chlorate, was shown to induce expression of NIAD, NIIA, and NRTA in A. nidulans and N. crassa (Crawford and Arst, 1993; Marzluf, 1997; Berger et al., 2006). Instead, we observed an expression of NIIA under nitrogen-starvation conditions and upon the addition of nitrate alone (Figure 3B). Despite the non-detectable transcript levels in response to nitrite in F. fujikuroi, at least low NiR enzyme levels must be present under this condition, because the fungus is clearly able to grow with nitrite as the sole nitrogen-source (Figure 1).

An explanation for the lack of nitrite-induced gene expression may be the toxicity of nitrite which makes it an even less favored nitrogen-source than nitrate. Because of this, many organisms actually have a tightly regulated nitrite uptake and export system to keep intracellular nitrite concentrations below toxic levels (Crawford and Arst, 1993; Lea et al., 2004; Wang et al., 2008; Jia et al., 2009; Cabrera et al., 2014). Taking this into consideration, it could be possible that extracellular nitrite induces only a slight or a significantly delayed expression of the nitrate assimilation system in F. fujikuroi, which was not detectable by Northern blot analysis. This would ensure that only limited amounts of nitrite are imported inside the cell, when they are absolutely necessary for growth. Additionally, the observed NIIA expression under nitrogen-starvation conditions would ensure that the NiR protein is already present inside the cell before the fungus comes into contact with nitrite as a potential nitrogen source. In this case, the toxic nitrite would be immediately metabolized to glutamine. The elucidation of these nitrite-specific responses in F. fujikuroi would be an interesting field for future studies.

\section{Sensing of Nitrate}

In F. fujikuroi, it was shown that the expression of many SM gene clusters is subject to NMR, including the GA and BIK biosynthesis genes (Candau et al., 1992; Mihlan et al., 2003; Wiemann et al., 2009, 2013; Tudzynski, 2014). The main effector mediating rapid and strong repression of these two SM clusters was shown to be glutamine, but a possible direct impact of nitrate on their expression needed to be investigated. In this study, we clearly showed that nitrate is not directly sensed as an effector of NMR, and rather that the repression of GA and BIK cluster genes in response to nitrate starts only after its conversion to ammonium and glutamine.

However, the fungus must be able to sense the presence of nitrate when preferred nitrogen sources are absent in order to quickly adapt to the changed conditions, e.g., by the nuclear translocation of NirA and subsequent transcriptional activation of the nitrate assimilation genes. The identification of a responsible nitrate sensor protein mediating the nitrate signal would be very intriguing. Research in A. nidulans has provided new insights regarding the signal transduction that leads to 
the nitrate-responsive nuclear retention of NirA, but the actual sensor still has not been identified (Bernreiter et al., 2007; Schinko et al., 2013; Gallmetzer et al., 2015).

Based on our results, the NR may play a possible nitrate sensing role based on the following reasoning. (1) The nitrate-dependent translocation of NirA was clearly disturbed in the $\triangle$ NIAD mutant (Figure 6). (2) The NR binds nitrate as a substrate, which might result in conformational changes and transduction of the signal to other proteins. The NR could therefore act as a nitrate sensor besides its enzymatic function. We recently described a similar dual function for the F. fujikuroi GS, which converts ammonium to glutamine. At the same time, the GS is involved in NMR-dependent regulation by transducing the presence of its substrate, ammonium, to other regulators (Wagner et al., 2013). However, even if the NR plays a role as a nitrate sensor, it cannot be the only one in F. fujikuroi because nitrate-induced gene expression was not disturbed in the $\triangle N I A D$ mutant.

Another interesting observation was that the nitrate-induced transcriptional activation of NIAD (Figure 3) and nuclear accumulation of NirA (Figure 6) occurred in the $\triangle N R T A$ mutant in a Wt-like manner. At first sight this may appear to be contradictory because our results identified NrtA as the main nitrate transporter in F. fujikuroi. However, this contradiction could be explained by three possibilities: (1) Traces of nitrate can be utilized without transporters by passive membrane permeation, (2) other transporters are able to transport low amounts of nitrate inside the cell, which are insufficient for growth but induce regulatory responses, and (3) nitrate is recognized before being internalized by an extracellular sensor protein. It should be noted that nitrate can also be generated inside the cell by oxidation of toxic nitrogenmonoxide (NO) radicals by flavohemoglobins (Schinko et al., 2010, 2013). However, this cannot be the explanation for the nitrate-induced effects we observed in $\triangle N R T A$, since they only occurred specifically after the addition of extracellular nitrate. In A. nidulans it was shown that the nitrate-induced translocation of NirA was significantly reduced in an AREA deletion strain due to the down-regulation of nitrate transporters in this strain. This would indicate that passive nitrate uptake via membrane permeation (Wang et al., 2008) is not sufficient for nitratemediated gene activation. Additionally, our NirA-localization studies showed that NirA-translocation in $\triangle N R T A$ takes place even when very low concentrations of nitrate are added to the cells, making a passive nitrate-uptake by membrane permeation less likely.

This leaves the more plausible possibilities of unspecific nitrate transport by other transporters and/or the presence of a sensor that recognizes extracellular nitrate. There has been no evidence for such a particular sensor in other organisms yet, but there are several examples were nutrient transporters have an additional sensing function, therefore known as transceptors (Lorenz and Heitman, 1998; Donaton et al., 2003; Biswas and Morschhäuser, 2005; Boeckstaens et al., 2008; Teichert et al., 2008; Rubio-Texeira et al., 2012; Van Zeebroeck et al., 2014). Possible candidates for unspecific nitrate transport or extracellular nitrate sensing in F. fujikuroi could be the previously mentioned nitrite transporter homolog (FFUJ_03993), which would be an interesting target for future characterization studies.

\section{CONCLUSION}

The results of our studies provide novel insights to the nitrate assimilation and sensing system in F. fujikuroi, uncovering similarities to other species but also some unique features. These findings contribute to the general understanding of alternate nitrogen assimilation pathways in fungal systems, which are connected to infection mechanisms of pathogens and regulation of SM production. We demonstrated that the genes NIAD, NIIA and NRTA encode the sole functional NR, NiR, and nitrate transporter in F. fujikuroi, respectively, and that their expression is induced by the presence of nitrate and repressed by high concentrations of the favored nitrogen-source glutamine, in a similar manner to the regulation-pattern in other organisms. In addition, both the pathway-specific TF NirA and the global, nitrogen-dependent regulator AreA act as positive regulators of the nitrate assimilation genes in F. fujikuroi, whereas AreB is not involved. While the expression of all genes depends clearly on NirA activity, AreA is essential for transcriptional activation of NIIA and NRTA, but surprisingly not for expression of NIAD. This indicates different regulatory mechanisms in this pathogen compared to other fungal species. In accordance with the findings in A. nidulans, NirA itself is regulated posttranslationally by a rapid, nitrate-induced nuclear accumulation and increasing levels of NirA protein, probably due to increased protein stability by nitrate. The nuclear translocation apparently depends on the NR, which indicates a potential dual role of the $\mathrm{NR}$ as a nitrate sensor. In contrast to nitrate, no nitrite-induced effects were observed on gene expression or NirA-translocation. Therefore, a more detailed study of the nitrite-specific transport and assimilation system in F. fujikuroi should be considered.

\section{MATERIALS AND METHODS}

\section{Fungal Strains and Culture Conditions}

In this study the following F. fujikuroi strains were used: Wt strain IMI58289 (Commonwealth Mycological Institute, Kew, UK), $\triangle A R E A-T 19$ (Tudzynski et al., 1999) and $\triangle A R E B-T 2.1$ (Michielse et al., 2014). Strains were maintained on solid CM (Pontecorvo et al., 1953) and cultivated at $28^{\circ} \mathrm{C}$ in darkness. Cultivation was performed as described in Wiemann et al. (2012), with liquid or solidified synthetic ICI (Imperial Chemical Industries, UK) minimal medium (Geissmann et al., 1966) supplemented with different nitrogen sources at $28^{\circ} \mathrm{C}$. For Northern blot analysis, the strains were cultivated in liquid ICI for $72 \mathrm{~h}$, harvested and mycelium was used for extraction of total RNA.

For yeast recombination cloning, Saccharomyces cerevisiae strain FGSC9721/FY834 (MATa his3 200 ura3-52 leu2 $\Delta 1$ lys2 $202 \operatorname{trpl} \Delta 63$ ) (Winston et al., 1995) was cultivated in $5 \mathrm{ml}$ liquid YPD ( $\mathrm{pH} 5.8,10 \mathrm{~g} / \mathrm{l}$ yeast extract, $20 \mathrm{~g} / \mathrm{l}$ Bacto-Trypton (Difco), $20 \mathrm{~g} / \mathrm{l}$ glucose) medium overnight at $200 \mathrm{rpm}$ and 
$30^{\circ} \mathrm{C}$. The culture was used to inoculate $50 \mathrm{ml}$ liquid YPD and incubated at $200 \mathrm{rpm}$ and $30^{\circ} \mathrm{C}$ for 4 to $6 \mathrm{~h}$ until an OD $600 \mathrm{~nm}$ of $\sim 1$ was reached. The harvested yeast cells were also used for yeast recombination cloning (Colot et al., 2006; Schumacher, 2012).

\section{Bacterial Strains and Plasmid Construction}

Escherichia coli strain Top10F' (Invitrogen, Groningen, Netherlands), cultivated in Lysogeny Broth medium (Sambrook et al., 1989) was used for plasmid propagation and amplification. For creation of the gene replacement vectors $\mathrm{p} \Delta$ NIAD, $\mathrm{p} \Delta$ NIIA, $\mathrm{p} \triangle \mathrm{NIRA}$, and $\mathrm{p} \triangle \mathrm{NRTA}$, flanking regions upstream $\left(5^{\prime}\right)$ and downstream $\left(3^{\prime}\right)$ of NIAD, NIIA, NIRA, and NRTA were amplified using the respective $5 \mathrm{~F} / 5 \mathrm{R}$ and $3 \mathrm{~F} / 3 \mathrm{R}$ primer pairs, while the hygromycin resistance cassette was amplified from pCSN44 (Staben et al., 1989) using the primer pair hphF/hphR (Supplementary Table S1). All three fragments were cloned into EcoRI/XhoI-restricted pRS426 (Christianson et al., 1992) by yeast recombinational cloning (see above). For transformation, the gene replacement fragments were amplified from the recombinated vectors by the respective primer pairs $5 \mathrm{~F} / 3 \mathrm{R}$ (Supplementary Table S1).

For generating the pOE::NrtA vector and the pOE::NirA::GFP fusion vector, full-length clones of NRTA and NIRA were amplified using the primer-combinations OE-NrtA-F/OE-NrtA-R and NIRA-GFP-F/NIRA-GFP-R, respectively, which contain overlapping sequences homologous to their destination vectors. The NIRA PCR product and the NcoI-digested plasmid pNAN-OGG (Schumacher, 2012), containing a nourseothricin resistance cassette, and a codonoptimized eGFP (Staben et al., 1989; Pöggeler et al., 2003) under control of the constitutive A. nidulans oliC promoter and the gluc terminator, were co-transformed into $S$. cerevisiae yielding pOE::NirA::GFP by yeast recombination cloning. In a similar manner the NRTA PCR product was transformed into vector pRS426 (Christianson et al., 1992), driven by the strong gpdA promoter of $A$. nidulans and a nourseothricin resistance cassette, yielding vector $\mathrm{pOE}:$ NrtA.

\section{Fungal Transformations}

Preparation of protoplasts of F. fujikuroi was carried out as described by Tudzynski et al. (1996). For deletion of NIAD, NIIA, NIRA, and NRTA, the F. fujikuroi Wt was transformed with gene replacement fragments derived from $\mathrm{p} \triangle$ NIAD, $\mathrm{p} \triangle$ NIIA, $\mathrm{p} \Delta$ NIRA, and $\mathrm{p} \Delta \mathrm{NRTA}$ (see above). Homologous gene-replacement was checked by diagnostic PCR with the respective primer pairs 5DIA-F/hphF, 3DIAR/hphR, and WT-F/WT-R (Supplementary Table S1). For expression of the NirA::GFP fusion construct, the strains $\triangle N I R A, \triangle N R T A, \triangle N I A D$, and $\triangle A R E A$ were transformed with $20 \mu \mathrm{g}$ of the pOE::NirA::GFP vector, yielding strains $\triangle N I R A-N I R A:: G F P, \triangle N R T A-N I R A:: G F P, \triangle N I A D-N I R A:: G F P$, and $\triangle A R E A-N I R A:: G F P$. For constitutive expression of $N R T A, 20 \mu \mathrm{g}$ of pOE::NrtA were transformed into $\triangle A R E A$, yielding strain $\triangle A R E A-O E:: N R T A$. Genomic integration of the overexpression-constructs was checked by diagnostic PCR with primers ogfp-seqR1/NIRA-WT-F and gpd-dia-for/NrtA-wt-R, respectively (Supplementary Table S1). Transformed protoplasts were regenerated at $28^{\circ} \mathrm{C}$ in a complete regeneration agar $(0.7 \mathrm{M}$ sucrose, $0.5 \mathrm{~g} / \mathrm{l}$ yeast extract) with $100 \mu \mathrm{g} / \mathrm{ml}$ nourseothricin (Werner Agents, Jena, Germany) or $100 \mu \mathrm{g} / \mathrm{ml}$ hygromycin (Sigma-Aldrich, Taufkirchen, Germany) for 4 to 7 days as specified above.

\section{PCR}

PCR reactions contained $25 \mathrm{ng}$ DNA, 5 pmol of each primer, $200 \mu \mathrm{M}$ desoxynucleotide tri-phosphates, 1 unit BioTherm DNA polymerase and $1 \mathrm{x}$ concentration of BioTherm buffer (Genecraft $\mathrm{GmbH}$, Lüdinghausen, Germany). The reactions were started with $4 \mathrm{~min}$ at $94^{\circ} \mathrm{C}$, followed by 35 cycles of $1 \mathrm{~min}$ per $\mathrm{kb}$ of the product at $94^{\circ} \mathrm{C}, 1 \mathrm{~min}$ at $56-65^{\circ} \mathrm{C}, 1 \mathrm{~min}$ at $70^{\circ} \mathrm{C}$, and a final $10 \mathrm{~min}$ at $70^{\circ} \mathrm{C}$. PCR products were cloned into pCR2.1-TOPO (Invitrogen). Resistance cassettes and eGFP for yeast recombination were amplified with the proofreading Phusion DNA polymerase (Finnzymes, Vantaa, Finland). These reactions contained $25 \mathrm{ng}$ DNA, 5 pmol of each primer, $200 \mu \mathrm{M}$ desoxynucleotide triphosphates, 1 unit Phusion polymerase and $1 \mathrm{x}$ concentration of HF-buffer. The reactions were started with $5 \mathrm{~min}$ at $95^{\circ} \mathrm{C}$, followed by 35 cycles of $1 \mathrm{~min}$ at $94^{\circ} \mathrm{C}, 1 \mathrm{~min}$ at $56-65^{\circ} \mathrm{C}, 1 \mathrm{~min}$ per $\mathrm{kb}$ of the product at $72^{\circ} \mathrm{C}$, and a final $10 \mathrm{~min}$ at $72^{\circ} \mathrm{C}$. All used primers are listed in Supplementary Table S1.

\section{Nucleic Acid Isolation and Northern Blot Analysis}

Lyophilized mycelium was ground into a fine powder and dispersed (in the case of DNA for use in PCR) in extraction buffer as described by Cenis (1992). Plasmid DNA was extracted using the Genomed plasmid extraction kit (Genomed, Löhne, Germany). Total F. fujikuroi RNA was isolated using the RNAgents total RNA isolation kit (Promega, Mannheim, Germany). For Northern blot analysis, samples of $20 \mu \mathrm{g}$ of total RNA were transferred to Hybond-N+ membranes after electrophoresis on a $1 \%(\mathrm{w} / \mathrm{v})$ agarose gel containing $1 \%(\mathrm{v} / \mathrm{v})$ formaldehyde. DNA probes were labeled with ${ }^{32} \mathrm{P}$ isotopes using the random oligomer-primer method (Sambrook et al., 1989). Hybridizations were carried out overnight at $65^{\circ} \mathrm{C}$ (Church and Gilbert, 1984). The following probes were used and amplified with the indicated primer combinations (see Supplementary Table S1 for primer sequences): BIK2 (bik2-F/bik2-R), $\quad C P S / K S \quad$ (cps/ks-RT-for/cps/ks-RT-rev), NIAD (NIAD-WT-F/NIAD-WT-R), NIIA (NIIA-WTF/NIIA-WT-R), NIRA (NIRA-WT-F/NIRA-WT-R), NRTA (NRTA-WT-F/NRTA-WT-R).

\section{Protein Isolation and Western Blot Analysis}

Total protein was extracted from freeze-dried mycelium as described before (Todd et al., 2005). Thirty micrograms of total protein extract were used per lane and separated by 
discontinuous SDS-polyacrylamide gel electrophoresis. The 5\% loading gel was adjusted to $\mathrm{pH} 6.8$, while the $8 \%$ separation gel was used at $\mathrm{pH}$ 8.8. The separation gel was semidry electro-blotted to a nitrocellulose membrane. For detection of the NirA::GFP fusion protein, rabbit polyclonal antibodies to Green Fluorescent Protein coupled to horseradish peroxidase (HRP) conjugates (Miltenyi Biotec GmbH, Bergisch Gladbach, Germany) were used for chemiluminescent detection of the proteins.

\section{Fluorescence Microscopy}

Ten microliters of F. fujikuroi mycelium, grown in liquid ICI media (with nitrogen source dependent on the experiment), was directly used for microscopy. GFP-fluorescence and Hoechst staining were observed using a Leica DMRBE microscope (Leica, Wetzlar, Germany) equipped with a high-performance charge-coupled device 12 bit SensiCam (PCO AG, Kehlheim, Germany) and filter set L5 (excitation band-pass filter 480/40, dichromatin mirror 505, suppression band-pass filter 527/30) for GFP. Nuclei were stained with Hoechst 33342 (Sigma-Aldrich, Chemie GmbH, Steinheim, Germany) in a 1:1000 dilution in McIlvaine Buffer pH 7.4 (Kangatharalingam and Ferguson, 1984) and visualized with filter set 49 DAPI shift free (excitation G 365, beam splitter FT 395, emission BP 445/50). Images (optical sections and Z-stacks) were captured with an AxioCam MRm camera and analyzed using the Axiovision Rel 4.8 software package (Zeiss, Germany).

\section{REFERENCES}

Arst, H. N., and Cove, D. J. (1973). Nitrogen metabolite repression in Aspergillus nidulans. Mol. Gen. Genet. 126, 111-141. doi: 10.1007/BF00330988

Aymoz, D., Wosika, V., Durandau, E., and Pelet, S. (2016). Real-time quantification of protein expression at the single-cell level via dynamic protein synthesis translocation reporters. Nat. Commun. 7:11304. doi: 10.1038/ncomms11304 doi: 10.1038/ncomms11304

Berger, H., Basheer, A., Böck, S., Reyes-Dominguez, Y., Dalik, T., Altmann, F., et al. (2008). Dissecting individual steps of nitrogen transcription factor cooperation in the Aspergillus nidulans nitrate cluster. Mol. Microbiol. 69, 1385-1398. doi: 10.1111/j.1365-2958.2008.06359.x

Berger, H., Pachlinger, R., Morozov, I., Goller, S., Narendja, F., Caddick, M., et al. (2006). The GATA factor AreA regulates localization and in vivo binding site occupancy of the nitrate activator NirA. Mol. Microbiol. 59, 433-446. doi: 10. 1111/j.1365-2958.2005.04957.x

Bernreiter, A., Ramon, A., Fernández-Martínez, J., Berger, H., Araújo-Bazan, L., Espeso, E. A., et al. (2007). Nuclear export of the transcription factor NirA is a regulatory checkpoint for nitrate induction in Aspergillus nidulans. Mol. Cell. Biol. 27, 791-802. doi: 10.1128/MCB.00761-06

Biswas, K., and Morschhäuser, J. (2005). The Mep2p ammonium permease controls nitrogen starvation-induced filamentous growth in Candida albicans. Mol. Microbiol. 56, 649-669. doi: 10.1111/j.1365-2958.2005.04576.x

Boeckstaens, M., André, B., and Marini, A. M. (2008). Distinct transport mechanisms in yeast ammonium transport/sensor proteins of the Mep/Amt/Rh family and impact on filamentation. J. Biol. Chem. 283, 21362-21370. doi: 10.1074/jbc.M801467200

Brownlee, A. G., and Arst, H. N. (1983). Nitrate uptake in Aspergillus nidulans and involvement of the third gene of the nitrate assimilation gene cluster. J. Bacteriol. 155, 1138-1146.

Burger, G., Strauss, J., Scazzocchio, C., and Lang, B. F. (1991). nirA, the pathwayspecific regulatory gene of nitrate assimilation in Aspergillus nidulans, encodes

\section{AUTHOR CONTRIBUTIONS}

$\mathrm{AP}$ and $\mathrm{BT}$ contributed to the design of the work. AP and JB were involved in data acquisition. AP, JB, and BT were involved in data analysis. AP and BT wrote the manuscript. All authors revised and approved the manuscript.

\section{FUNDING}

This project was funded by the German Research Foundation (Deutsche Forschungsgemeinschaft, DFG), grant number TU101/12-3.

\section{ACKNOWLEDGMENTS}

The authors would like to thank Kathleen Huß for creation of the $\triangle N I R A$ and $\triangle N R T A$ mutants. We are particularly grateful to Brian Williamson for critical reading of this manuscript.

\section{SUPPLEMENTARY MATERIAL}

The Supplementary Material for this article can be found online at: http://journal.frontiersin.org/article/10.3389/fmicb. 2017.00381/full\#supplementary-material

a putative GAL4-type zinc finger protein and contains four introns in highly conserved regions. Mol. Cell. Biol. 11, 5746-5755. doi: 10.1128/MCB.11.11.5746 Cabrera, E., González-Montelongo, R., Giraldez, T., Alvarez de la Rosa, D., and Siverio, J. M. (2014). Molecular components of nitrate and nitrite efflux in yeast. Eukaryot. Cell 13, 267-278. doi: 10.1128/EC.00268-13

Caddick, M. X., Jones, M. G., van Tonder, J. M., Le Cordier, H., Narendja, F., Strauss, J., et al. (2006). Opposing signals differentially regulate transcript stability in Aspergillus nidulans. Mol. Microbiol. 62, 509-519. doi: 10.1111/j. 1365-2958.2006.05383.x

Campbell, W. H., and Kinghorn, J. R. (1990). Functional domains of assimilatory nitrate reductases and nitrite reductases. Trends Biochem. Sci. 15, 315-319. doi: 10.1016/0968-0004(90)90021-3

Candau, R., Avalos, J., and Cerdá-Olmedo, E. (1992). Regulation of gibberellin biosynthesis in Gibberella fujikuroi. Plant Physiol. 100, 1184-1188. doi: 10.1104/ pp.100.3.1184

Cenis, J. L. (1992). Rapid extraction of fungal DNA for PCR amplification. Nucleic Acids Res. 20, 2380. doi: 10.1093/nar/20.9.2380

Christianson, T. W., Sikorski, R. S., Dante, M., Shero, J. H., and Hieter, P. (1992). Multifunctional yeast high-copy-number shuttle vectors. Gene 110, 119-122. doi: 10.1016/0378-1119(92)90454-W

Church, G. M., and Gilbert, W. (1984). Genomic sequencing. Proc. Natl. Acad. Sci. U.S.A. 81, 1991-1995. doi: 10.1073/pnas.81.7.1991

Colot, H. V., Park, G., Turner, G. E., Ringelberg, C., Crew, C. M., Litvinkova, L., et al. (2006). A high-throughput gene knockout procedure for Neurospora reveals functions for multiple transcription factors. Proc. Natl. Acad. Sci. U.S.A. 103, 10352-10357. doi: 10.1073/pnas.0601456103

Cove, D. J. (1976). Cholorate toxicity in Aspergillus nidulans: the selection and characterisation of chlorate resistant mutants. Heredity 36, 191-203. doi: 10. 1038/hdy.1976.24

Cove, D. J. (1979). Genetic studies of nitrate assimilation in Aspergillus nidulans. Biol. Rev. Camb. Philos. Soc. 54, 291-327. doi: 10.1111/j.1469-185X.1979. tb01014.x 
Crawford, N. M., and Arst, H. N. (1993). The molecular genetics of nitrate assimilation in fungi and plants. Annu. Rev. Genet. 27, 115-146. doi: 10.1146/ annurev.ge.27.120193.000555

Donaton, M. C. V., Holsbeeks, I., Lagatie, O., Van Zeebroeck, G., Crauwels, M., Winderickx, J., et al. (2003). The Gap1 general amino acid permease acts as an amino acid sensor for activation of protein kinase A targets in the yeast Saccharomyces cerevisiae. Mol. Microbiol. 50, 911-929. doi: 10.1046/j.13652958.2003.03732.x

Dunn-Coleman, N. S., Tomsett, A. B., and Garrett, R. H. (1981). The regulation of nitrate assimilation in Neurospora crassa: biochemical analysis of the nmr-1 mutants. Mol. Gen. Genet. 182, 234-239. doi: 10.1007/BF00269663

Dzikowska, A., Kacprzak, M., Tomecki, R., Koper, M., Scazzocchio, C., and Weglenski, P. (2003). Specific induction and carbon/nitrogen repression of arginine catabolism gene of Aspergillus nidulans-functional in vivo analysis of the otaA promoter. Fungal Genet. Biol. 38, 175-186. doi: 10.1016/S10871845(02)00522-4

Feng, B., and Marzluf, G. A. (1998). Interaction between major nitrogen regulatory protein NIT2 and pathway-specific regulatory factor NIT4 is required for their synergistic activation of gene expression in Neurospora crassa. Mol. Cell. Biol. 18, 3983-3990. doi: 10.1128/MCB.18.7.3983

Gallmetzer, A., Silvestrini, L., Schinko, T., Gesslbauer, B., Hortschansky, P., Dattenböck, C., et al. (2015). Reversible oxidation of a conserved methionine in the nuclear export sequence determines subcellular distribution and activity of the fungal nitrate regulator NirA. PLoS Genet. 11:e1005297. doi: 10.1371/ journal.pgen.1005297

Geissmann, T. A., Verbiscar, A. J., Phinney, B. O., and Cragg, G. (1966). Studies on the biosynthesis of gibberellins from (-)-kaurenoic acid in cultures of Gibberella fujikuroi. Phytochemistry 5, 933-947. doi: 10.1016/S0031-9422(00)82790-9

Gorfer, M., Blumhoff, M., Klaubauf, S., Urban, A., Inselsbacher, E., Bandian, D., et al. (2011). Community profiling and gene expression of fungal assimilatory nitrate reductases in agricultural soil. ISME J. 5, 1771-1783. doi: 10.1038/ismej. 2011.53

Haas, H., Angermayr, K., Zadra, I., and Stöffler, G. (1997). Overexpression of nreB, a new GATA factor-encoding gene of Penicillium chrysogenum, leads to repression of the nitrate assimilatory gene cluster. J. Biol. Chem. 272, 22576-22582. doi: 10.1074/jbc.272.36.22576

Jia, W., Tovell, N., Clegg, S., Trimmer, M., and Cole, J. (2009). A single channel for nitrate uptake, nitrite export and nitrite uptake by Escherichia coli NarU and a role for NirC in nitrite export and uptake. Biochem. J. 417, 297-304. doi: 10.1042/BJ20080746

Johnstone, I. L., McCabe, P. C., Greaves, P., Gurr, S. J., Cole, G. E., Brow, M. A., et al. (1990). Isolation and characterisation of the crnA-niiA-niaD gene cluster for nitrate assimilation in Aspergillus nidulans. Gene 90, 181-192. doi: 10.1016/ 0378-1119(90)90178-T

Kangatharalingam, N., and Ferguson, M. W. (1984). A simple and rapid technique for fluorescence staining of fungal nuclei. Curr. Microbiol. 10, 99-103. doi: 10.1007/BF01575767

Kudla, B., Caddick, M. X., Langdon, T., Martinez-Rossi, N. M., Bennett, C. F., Sibley, S., et al. (1990). The regulatory gene areA mediating nitrogen metabolite repression in Aspergillus nidulans. Mutations affecting specificity of gene activation alter a loop residue of a putative zinc finger. EMBO J. 9, 1355-1364.

Lea, U. S., Ten Hoopen, F., Provan, F., Kaiser, W. M., Meyer, C., and Lillo, C. (2004). Mutation of the regulatory phosphorylation site of tobacco nitrate reductase results in high nitrite excretion and $\mathrm{NO}$ emission from leaf and root tissue. Planta 219, 59-65. doi: 10.1007/s00425-0041209-6

Leach, M. D., and Brown, A. J. P. (2012). Posttranslational modifications of proteins in the pathobiology of medically relevant fungi. Eukaryot. Cell 11, 98-108. doi: 10.1128/EC.05238-11

López-Berges, M. S., Rispail, N., Prados-Rosales, R. C., and Pietro, A. D. (2010). A nitrogen response pathway regulates virulence functions in Fusarium oxysporum via the protein kinase TOR and the bZIP protein MeaB. Plant Cell 22, 2459-2475. doi: 10.1105/tpc.110.075937

López-Berges, M. S., Schäfer, K., Hera, C., and Di Pietro, A. (2014). Combinatorial function of velvet and AreA in transcriptional regulation of nitrate utilization and secondary metabolism. Fungal Genet. Biol. 62, 78-84. doi: 10.1016/j.fgb. 2013.11.002
Lorenz, M. C., and Heitman, J. (1998). The MEP2 ammonium permease regulates pseudohyphal differentiation in Saccharomyces cerevisiae. EMBO J. 17, 12361247. doi: $10.1093 /$ emboj/17.5.1236

Macios, M., Caddick, M. X., Weglenski, P., Scazzocchio, C., and Dzikowska, A. (2012). The GATA factors AREA and AREB together with the co-repressor NMRA, negatively regulate arginine catabolism in Aspergillus nidulans in response to nitrogen and carbon source. Fungal Genet. Biol. 49, 189-198. doi: 10.1016/j.fgb.2012.01.004

Magasanik, B., and Kaiser, C. A. (2002). Nitrogen regulation in Saccharomyces cerevisiae. Gene 290, 1-18. doi: 10.1016/S0378-1119(02)00558-9

Malardier, L., Daboussi, M. J., Julien, J., Roussel, F., Scazzocchio, C., and Brygoo, Y. (1989). Cloning of the nitrate reductase gene (niaD) of Aspergillus nidulans and its use for transformation of Fusarium oxysporum. Gene 78, 147-156. doi: 10.1016/0378-1119(89)90322-3

Marchive, C., Roudier, F., Castaings, L., Bréhaut, V., Blondet, E., Colot, V., et al. (2013). Nuclear retention of the transcription factor NLP7 orchestrates the early response to nitrate in plants. Nat. Commun. 4:1713. doi: 10.1038/ncomms 2650

Marzluf, G. A. (1997). Genetic regulation of nitrogen metabolism in the fungi. Microbiol. Mol. Biol. Rev. 61, 17-32.

Michielse, C. B., Pfannmüller, A., Macios, M., Rengers, P., Dzikowska, A., and Tudzynski, B. (2014). The interplay between the GATA transcription factors AreA, the global nitrogen regulator and AreB in Fusarium fujikuroi. Mol. Microbiol. 91, 472-493. doi: 10.1111/mmi.12472

Mihlan, M., Homann, V., Liu, T.-W. D., and Tudzynski, B. (2003). AREA directly mediates nitrogen regulation of gibberellin biosynthesis in Gibberella fujikuroi, but its activity is not affected by NMR. Mol. Microbiol. 47, 975-991. doi: 10. 1046/j.1365-2958.2003.03326.x

Morozov, I. Y., Galbis-Martinez, M., Jones, M. G., and Caddick, M. X. (2001). Characterization of nitrogen metabolite signalling in Aspergillus via the regulated degradation of areA mRNA. Mol. Microbiol. 42, 269-277. doi: 10. 1046/j.1365-2958.2001.02636.x

Morozov, I. Y., Martinez, M. G., Jones, M. G., and Caddick, M. X. (2000). A defined sequence within the $3^{\prime}$ UTR of the areA transcript is sufficient to mediate nitrogen metabolite signalling via accelerated deadenylation. Mol. Microbiol. 37, 1248-1257. doi: 10.1046/j.1365-2958.2000.02085.x

Muro-Pastor, M. I., Gonzalez, R., Strauss, J., Narendja, F., and Scazzocchio, C. (1999). The GATA factor AreA is essential for chromatin remodelling in a eukaryotic bidirectional promoter. EMBO J. 18, 1584-1597. doi: 10.1093/ emboj/18.6.1584

Muro-Pastor, M. I., Strauss, J., Ramón, A., and Scazzocchio, C. (2004). A paradoxical mutant GATA factor. Eukaryot. Cell 3, 393-405. doi: 10.1128/ EC.3.2.393-405.2004

Narendja, F., Goller, S. P., Wolschek, M., and Strauss, J. (2002). Nitrate and the GATA factor AreA are necessary for in vivo binding of NirA, the pathwayspecific transcriptional activator of Aspergillus nidulans. Mol. Microbiol. 44, 573-583. doi: 10.1046/j.1365-2958.2002.02911.x

Pfannmüller, A., Wagner, D., Sieber, C., Schönig, B., Boeckstaens, M., Marini, A. M., et al. (2015). The general amino acid permease Ffgap1 of Fusarium fujikuroi is sorted to the vacuole in a nitrogen-dependent, but Npr1 kinaseindependent manner. PLOS ONE 10:e0125487. doi: 10.1371/journal.pone. 0125487

Platt, A., Langdon, T., Arst, H. N., Kirk, D., Tollervey, D., Sanchez, J. M., et al. (1996). Nitrogen metabolite signalling involves the C-terminus and the GATA domain of the Aspergillus transcription factor AREA and the 3' untranslated region of its mRNA. EMBO J. 15, 2791-2801.

Pöggeler, S., Masloff, S., Hoff, B., Mayrhofer, S., and Kück, U. (2003). Versatile EGFP reporter plasmids for cellular localization of recombinant gene products in filamentous fungi. Curr. Genet. 43, 54-61. doi: 10.1007/s00294-003-0370-y

Pontecorvo, G., Roper, J. A., Hemmons, L. M., Macdonald, K. D., and Bufton, A. W. J. (1953). The genetics of Aspergillus nidulans. Adv. Genet. 5, 141-238. doi: 10.1016/s0065-2660(08)60408-3

Premakumar, R., Sorger, G. J., and Gooden, D. (1979). Nitrogen metabolite repression of nitrate reductase in Neurospora crassa. J. Bacteriol. 137, 1119-1126.

Punt, P. J., Strauss, J., Smit, R., Kinghorn, J. R., van den Hondel, C. A., and Scazzocchio, C. (1995). The intergenic region between the divergently transcribed niiA and niaD genes of Aspergillus nidulans contains multiple 
NirA binding sites which act bidirectionally. Mol. Cell. Biol. 15, 5688-5699. doi: 10.1128/MCB.15.10.5688

Rubio-Texeira, M., Van Zeebroeck, G., and Thevelein, J. M. (2012). Peptides induce persistent signaling from endosomes by a nutrient transceptor. Nat. Chem. Biol. 8, 400-408. doi: 10.1038/nchembio.910

Sambrook, J., Fritsch, E. F., and Maniatis, T. (1989). Molecular Cloning: A Laboratory Manual, 2nd Edn. Cold Spring Harbor, NY: Cold Spring Harbor Laboratory.

Scazzocchio, C., and Arst, H. N. Jr. (1989). "Regulation of nitrate assimilation in Aspergillus nidulans," in Molecular and Genetic Aspects of Nitrate Assimilation, eds J. L. Wray and J. R. Kinghorn (Oxford: Oxford University Press), 299-313.

Schinko, T., Berger, H., Lee, W., Gallmetzer, A., Pirker, K., Pachlinger, R., et al. (2010). Transcriptome analysis of nitrate assimilation in Aspergillus nidulans reveals connections to nitric oxide metabolism. Mol. Microbiol. 78, 720-738. doi: $10.1111 /$ j.1365-2958.2010.07363.x

Schinko, T., Gallmetzer, A., Amillis, S., and Strauss, J. (2013). Pseudo-constitutivity of nitrate-responsive genes in nitrate reductase mutants. Fungal Genet. Biol. 54, 34-41. doi: 10.1016/j.fgb.2013.02.003

Schumacher, J. (2012). Tools for Botrytis cinerea: new expression vectors make the gray mold fungus more accessible to cell biology approaches. Fungal Genet. Biol. 49, 483-497. doi: 10.1016/j.fgb.2012.03.005

Siverio, J. M. (2002). Assimilation of nitrate by yeasts. FEMS Microbiol. Rev. 26, 277-284. doi: 10.1111/j.1574-6976.2002.tb00615.x

Song, M., Xu, X., Hu, Q., Tian, Y., Ouyang, H., and Zhou, C. (2007). Interactions of plant species mediated plant competition for inorganic nitrogen with soil microorganisms in an alpine meadow. Plant Soil 297, 127-137. doi: 10.1007/ s11104-007-9326-1

Staben, C., Jensen, B., Singer, M., Pollock, J., Schechtman, M., and Kinsey, J. (1989). Use of a bacterial hygromycin B resistance gene as a dominant selectable marker in Neurospora crassa transformation. Fungal Genet. Newsl. 36, 79-81.

Strauss, J., Muro-Pastor, M. I., and Scazzocchio, C. (1998). The regulator of nitrate assimilation in ascomycetes is a dimer which binds a nonrepeated, asymmetrical sequence. Mol. Cell. Biol. 18, 1339-1348. doi: 10.1128/MCB.18.3.1339

Tao, Y., and Marzluf, G. A. (1999). The NIT2 nitrogen regulatory protein of Neurospora: expression and stability of nit-2 mRNA and protein. Curr. Genet. 36, 153-158. doi: 10.1007/s002940050485

Teichert, S., Rutherford, J. C., Wottawa, M., Heitman, J., and Tudzynski, B. (2008). Impact of ammonium permeases MepA, MepB, and MepC on nitrogenregulated secondary metabolism in Fusarium fujikuroi. Eukaryot. Cell 7, 187-201. doi: 10.1128/EC.00351-07

Todd, R. B., Fraser, J. A., Wong, K. H., Davis, M. A., and Hynes, M. J. (2005). Nuclear accumulation of the GATA factor AreA in response to complete nitrogen starvation by regulation of nuclear export. Eukaryot. Cell 4, 1646-1653. doi: 10.1128/EC.4.10.1646-1653.2005

Tudzynski, B. (2014). Nitrogen regulation of fungal secondary metabolism in fungi. Front. Microbiol. 5:656. doi: 10.3389/fmicb.2014.00656

Tudzynski, B., Homann, V., Feng, B., and Marzluf, G. A. (1999). Isolation, characterization and disruption of the areA nitrogen regulatory gene of Gibberella fujikuroi. Mol. Gen. Genet. 261, 106-114. doi: 10.1007/ s004380050947

Tudzynski, B., Mende, K., Weltring, K. M., Kinghorn, J. R., and Unkles, S. E. (1996). The Gibberella fujikuroi niaD gene encoding nitrate reductase: isolation, sequence, homologous transformation and electrophoretic karyotype location. Microbiology 142(Pt 3), 533-539. doi: 10.1099/13500872-1423-533

Unkles, S. E., Hawker, K. L., Grieve, C., Campbell, E. I., Montague, P., and Kinghorn, J. R. (1991). crnA encodes a nitrate transporter in Aspergillus nidulans. Proc. Natl. Acad. Sci. U.S.A. 88, 204-208. doi: 10.1073/pnas.88.1.204
Unkles, S. E., Zhou, D., Siddiqi, M. Y., Kinghorn, J. R., and Glass, A. D. (2001). Apparent genetic redundancy facilitates ecological plasticity for nitrate transport. EMBO J. 20, 6246-6255. doi: 10.1093/emboj/20.22.6246

van Wijk, D. J., Kroon, S. G., and Garttener-Arends, I. C. (1998). Toxicity of chlorate and chlorite to selected species of algae, bacteria, and fungi. Ecotoxicol. Environ. Saf. 40, 206-211. doi: 10.1006/eesa.1998.1685

Van Zeebroeck, G., Rubio-Texeira, M., Schothorst, J., and Thevelein, J. M. (2014). Specific analogues uncouple transport, signalling, oligo-ubiquitination and endocytosis in the yeast Gap1 amino acid transceptor. Mol. Microbiol. 93, 213-233. doi: $10.1111 / \mathrm{mmi} .12654$

Wagner, D., Wiemann, P., Huß, K., Brandt, U., Fleißner, A., and Tudzynski, B. (2013). A sensing role of the glutamine synthetase in the nitrogen regulation network in Fusarium fujikuroi. PLoS ONE 8:e80740. doi: 10.1371/journal.pone. 0080740

Wang, Y., Li, W., Siddiqi, Y., Symington, V. F., Kinghorn, J. R., Unkles, S. E., et al. (2008). Nitrite transport is mediated by the nitrite-specific high-affinity NitA transporter and by nitrate transporters NrtA, NrtB in Aspergillus nidulans. Fungal Genet. Biol. 45, 94-102. doi: 10.1016/j.fgb.2007.10.001

Wiame, J. M., Grenson, M., and Arst, H. N. (1985). Nitrogen catabolite repression in yeasts and filamentous fungi. Adv. Microb. Physiol. 26, 1-88. doi: 10.1016/ S0065-2911(08)60394-X

Wiemann, P., Albermann, S., Niehaus, E.-M., Studt, L., von Bargen, K. W., Brock, N. L., et al. (2012). The Sfp-type 4'-phosphopantetheinyl transferase Pptl of Fusarium fujikuroi controls development, secondary metabolism and pathogenicity. PLOS ONE 7:e37519. doi: 10.1371/journal.pone.0037519

Wiemann, P., Sieber, C. M. K., von Bargen, K. W., Studt, L., Niehaus, E.-M., Espino, J. J., et al. (2013). Deciphering the cryptic genome: genome-wide analyses of the rice pathogen Fusarium fujikuroi reveal complex regulation of secondary metabolism and novel metabolites. PLoS Pathog. 9:e1003475. doi: 10.1371/ journal.ppat.1003475

Wiemann, P., Willmann, A., Straeten, M., Kleigrewe, K., Beyer, M., Humpf, H.-U., et al. (2009). Biosynthesis of the red pigment bikaverin in Fusarium fujikuroi: genes, their function and regulation. Mol. Microbiol. 72, 931-946. doi: 10.1111/ j.1365-2958.2009.06695.x

Winston, F., Dollard, C., and Ricupero-Hovasse, S. L. (1995). Construction of a set of convenient Saccharomyces cerevisiae strains that are isogenic to S288C. Yeast 11, 53-55. doi: 10.1002/yea.320110107

Wodzinski, R. S., Labeda, D. P., and Alexander, M. (1978). Effects of low concentrations of bisulfite-sulfite and nitrite on microorganisms. Appl. Environ. Microbiol. 35, 718-723.

Wong, K. H., Hynes, M. J., Todd, R. B., and Davis, M. A. (2009). Deletion and overexpression of the Aspergillus nidulans GATA factor AreB reveals unexpected pleiotropy. Microbiology 155, 3868-3880. doi: 10.1099/mic.0. 031252-0

Yuan, G. F., Fu, Y. H., and Marzluf, G. A. (1991). nit-4, a pathway-specific regulatory gene of Neurospora crassa, encodes a protein with a putative binuclear zinc DNA-binding domain. Mol. Cell. Biol. 11, 5735-5745. doi: 10. 1128/MCB.11.11.5735

Conflict of Interest Statement: The authors declare that the research was conducted in the absence of any commercial or financial relationships that could be construed as a potential conflict of interest.

Copyright (C) 2017 Pfannmüller, Boysen and Tudzynski. This is an open-access article distributed under the terms of the Creative Commons Attribution License (CC BY). The use, distribution or reproduction in other forums is permitted, provided the original author(s) or licensor are credited and that the original publication in this journal is cited, in accordance with accepted academic practice. No use, distribution or reproduction is permitted which does not comply with these terms. 